\title{
The Complexity of Lattice-Based Fuzzy Description Logics
}

\author{
From $\mathcal{A L C}$ to $\mathcal{S H \mathcal { H }}$
}

\author{
Stefan Borgwardt · Rafael Peñaloza
}

Received: 25 January 2012 / Revised: 2 October 2012 / Accepted: 11 October 2012 / Published online: 27 November 2012

(C) Springer-Verlag Berlin Heidelberg 2012

\begin{abstract}
We study the complexity of reasoning in fuzzy description logics with semantics based on finite residuated

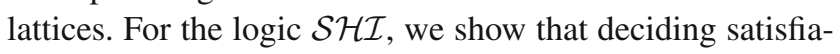
bility and subsumption of concepts, with or without a TBox, are EXPTIME-complete problems. In $\mathcal{A L C H} \mathcal{I}$ and a variant of $\mathcal{S I}$, these decision problems become PSPACE-complete when restricted to acyclic TBoxes. This matches the known complexity bounds for reasoning in crisp description logics between $\mathcal{A L C}$ and $\mathcal{S H} \mathcal{I}$.
\end{abstract}

Keywords Knowledge representation - Description logics · Mathematical fuzzy logic $\cdot$ Residuated lattices . Complexity analysis $\cdot$ Tree automata

\section{Introduction}

To enable automatic systems to draw inferences about a situation, the knowledge of the application domain is often represented in a logical calculus. Description Logics (DLs) [1] are a family of such logical formalisms that describe knowledge with the help of concepts (unary predicates) and roles (binary predicates). For example, the concept Human can be used to model the set of all human beings and the role hasChild to express the parentage relationship between individual humans.

S. Borgwardt is partially supported by the DFG under grant BA 1122/17-1.

\section{S. Borgwardt $(\varangle) \cdot$ R. Peñaloza}

Institute for Theoretical Computer Science,

Technische Universität Dresden,

01062 Dresden, Germany

e-mail: stefborg@tcs.inf.tu-dresden.de

R. Peñaloza

e-mail: penaloza@tcs.inf.tu-dresden.de
Complex concepts can then be built using logical constructors such as conjunction ( $\square)$ and existential restriction $(\exists)$. For example, one can express the set of all parents through the concept Human $\sqcap \exists$ hasChild.Human, i.e. the set of all humans that have at least one child that is also human.

Description logics are determined by the set of constructors that they allow. The smallest propositionally closed DL is called $\mathcal{A L C}$. The logic that extends $\mathcal{A} \mathcal{L C}$ with transitive roles is denoted by $\mathcal{S}$. Two other important constructors are inverse roles and role hierarchies; logics including these constructors bear the letters $\mathcal{I}$ and $\mathcal{H}$, respectively, in their names. Thus, $\mathcal{S H \mathcal { I }}$ is the DL that extends $\mathcal{A} \mathcal{L C}$ with transitive and inverse roles, and role hierarchies. The relative expressivity of these logics is depicted in Fig. 1.

In description logics, knowledge is represented through a set of axioms, called an ontology, that restrict the class of possible interpretations. It is possible, for example, to express that every person having a child must be a parent with the axiom $\exists$ hasChild. Human $\sqsubseteq$ Parent. Two of the main reasoning tasks in DLs are deciding subsumption (is concept $C$ always included in concept $D$ ?) and satisfiability (is concept $C$ non-contradictory?). The precise complexity of answering these and similar questions is one of the major issues in DL research.

DLs have been successfully applied to represent knowledge, in particular in the biomedical domain, where very large ontologies such as SNOMEDCT ${ }^{1}$ and Galen ${ }^{2}$ are used in practice. Moreover, the current standard ontology language for the semantic web, OWL $2,{ }^{3}$ is based on the DL $\mathcal{S R O I} \mathcal{Q}(D)$, whose expressivity goes beyond $\mathcal{S H I}$.

\footnotetext{
$\overline{1}$ http://www.ihtsdo.org/snomed-ct/.

2 http://www.opengalen.org/.

${ }^{3}$ http://www.w3.org/TR/owl2-overview/.
} 
The traditional way to interpret description logic concepts as subsets of a domain is often ill-suited to express vague or imprecise concepts like HighTemperature: there is no clearcut point where a temperature becomes "high," but we know that $36^{\circ} \mathrm{C}$ should belong to this concept to a "lower degree" than, say $39^{\circ} \mathrm{C}$. This poses a big problem for the use of medical ontologies, where most of the observations are necessarily vague. Fuzzy Description Logics have been introduced to alleviate this problem by allowing an element to belong to a concept with a certain membership degree. The underlying notions are adapted from Fuzzy Set Theory [33] and Mathematical Fuzzy Logic [22]. A more detailed description on the applicability of fuzzy semantics for medical applications can be found in [27].

Fuzzy extensions of DLs have been studied for more than a decade, and the literature on the topic is very extensive (see [26] for a survey). However, most of those approaches are based on the simple Zadeh semantics with truth values ranging over the interval $[0,1]$ of rational numbers, where conjunction is interpreted as the minimum. The last lustrum has seen a shift towards more general semantics for the treatment of vagueness. On the one hand, the use of continuous $t$-norms as the underlying interpretation function for conjunction was proposed in [23]. On the other hand, [30] allows lattice-based truth values, but still restricts to Zadeh-like semantics.

Most of the work since then has focused on $t$-norm based semantics over the unit interval; yet, ontologies are usually restricted to be unfoldable or acyclic $[6,8,9]$. Indeed, we have shown that removing this restriction easily leads to undecidability. If general concept inclusion axioms (GCIs) are allowed, then we have shown that reasoning in $\mathcal{A L C}$ over infinite lattices becomes undecidable [15]. Similar results have been shown for several fuzzy DLs based on $\mathcal{A L C}$ with $t$-norms over the unit interval $[3,4,16,18]$. These results motivate restricting the logics, e.g. to finitely valued semantics.

If one considers the Lukasiewicz $t$-norm over finitely many values, then reasoning is decidable even for very expressive DLs, as shown in [11] through a reduction to crisp reasoning. When restricted to $\mathcal{A L C}$ without terminological axioms, concept satisfiability is PSPACE-complete as in the crisp case [17]. ${ }^{4}$

When the underlying semantics is generalized to lattices, we denote the resulting DLs with the prefix $L$-, for example $L-\mathcal{A L C}$. For $L-\mathcal{A L C}$ with general TBoxes, reasoning is EXPTIME-complete [12,14], again matching the complexity of the crisp case, even if arbitrary (finite) lattices and $t$-norms are allowed. However, the complexity of subsumption of concepts has not yet been studied, since the standard reduction

\footnotetext{
4 The paper [17] actually considers the modal logic K, but the results can be easily transferred to $\mathcal{A} \mathcal{L C}$.
}

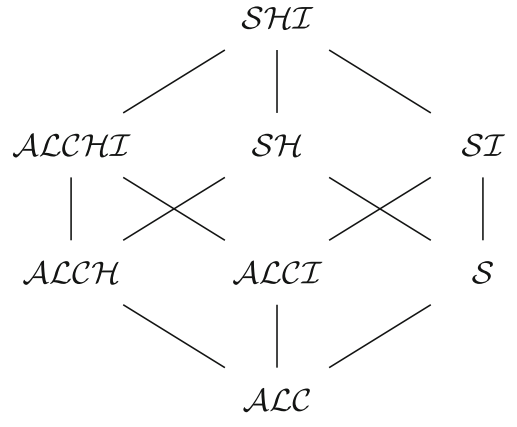

Fig. 1 The relative expressivity of the description logics between $\mathcal{A L C}$ and $\mathcal{S H \mathcal { I }}$

used in crisp DLs does not work with general $t$-norm semantics.

In [13], these results were generalized to the fuzzy logic $L-\mathcal{A L C I}$. Concept satisfiability in this logic is EXPTIMEcomplete w.r.t. general TBoxes, and PSPACE-complete w.r.t. acyclic TBoxes. Moreover, the same complexity bounds are also shown to hold for deciding subsumption between concepts.

In this paper, we extend the results from [13] and analyze the complexity of reasoning in the fuzzy description logics between $L-\mathcal{A L C}$ and $L-\mathcal{S H} \mathcal{H}$ (see Fig. 1). In most cases, the complexity bounds of the crisp case can be matched. More precisely, we show that the following complexity results for crisp DLs also hold for semantics based on finite residuated lattices:

1. satisfiability and subsumption w.r.t. general TBoxes is EXPTIME-complete in every DL between $L-\mathcal{A L C}$ and $L-\mathcal{S H} \mathcal{I}$

2. reasoning w.r.t. acyclic TBoxes is PSPACE-complete in

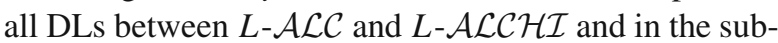
logic $L-\mathcal{S} \mathcal{I}_{\mathrm{C}}$ of $L-\mathcal{S I} ;$

3. even if the TBox is empty, reasoning in the logics $L-\mathcal{S H}$ and $L-\mathcal{S H} \mathcal{I}$ is EXPTIME-complete.

All the lower bounds are given by the complexity results for crisp DLs. To prove 1. and 3., we provide an automatabased EXPTIME reasoning procedure for $L-\mathcal{S H I}$ w.r.t. general TBoxes (Sect. 4). For 2., we show in Sect. 5 that the automata can be modified into a so-called PSPACE-on-the-fly construction when restricted to the logics $L-\mathcal{S} \mathcal{I}_{\mathrm{C}}$ and $L-\mathcal{A L C H \mathcal { I }}$, thus obtaining the PSPACE upper bound. The precise complexity of full $L-\mathcal{S I}$ is left open.

To the best of our knowledge, the precise complexity of (crisp) $\mathcal{A L C H} \mathcal{H}$ has not yet been studied. Thus, our analysis of the complexity of fuzzy DLs also yields a new, although not surprising, complexity result for this crisp DL.

To improve the readability of this paper, some of the more technical proofs have been moved to an appendix.

\footnotetext{
${ }^{5}$ In $L-\mathcal{S} \mathcal{I}_{\mathrm{C}}$, roles are restricted to be crisp
} 


\section{Preliminaries}

We first recall some results from automata theory that will allow us to obtain tight upper bounds for the complexity of reasoning in some special fuzzy DLs. The full proofs and main intuitions behind these results can be found in [2]. To aid the understanding of the notions introduced, we include a small example in the appendix (Example B.1).

We then provide a short introduction to residuated lattices, which are the basis for the semantics of our logic. For a more comprehensive view on this topic, we refer the reader to $[19,21]$.

\subsection{PSPACE Automata}

We will obtain the main results through a reduction to the emptiness problem of looping automata on infinite trees (see Sect. 4). These automata receive as input the (unlabeled) infinite $k$-ary tree with $k \in \mathbb{N}$. The nodes of this tree are words in $K^{*}$, where $K:=\{1, \ldots, k\}$ : the empty word $\varepsilon$ represents the root node, and $u i$ represents the $i$ th successor of the node $u$. A path is a sequence $v_{1}, \ldots, v_{m}$ of nodes such that $v_{1}=\varepsilon$ and $v_{i+1}$ is a successor of $v_{i}$ for every $i \geq 1$.

Definition 2.1 (looping automaton) A looping automaton (LA) is a tuple $\mathcal{A}=(Q, I, \Delta)$ where $Q$ is a finite set of states, $I \subseteq Q$ a set of initial states, and $\Delta \subseteq Q^{k+1}$ the transition relation. A run of $\mathcal{A}$ is a mapping $r: K^{*} \rightarrow Q$ assigning states to each node of $K^{*}$ such that $r(\varepsilon) \in I$ and for every $u \in K^{*},(r(u), r(u 1), \ldots, r(u k)) \in \Delta$. The emptiness problem for looping automata is to decide whether a given looping automaton has a run.

The emptiness of looping automata can be decided in (deterministic) polynomial time using a bottom-up approach that finds all the states that can appear in a run [32]. In some cases, it is convenient to use a non-deterministic top-down approach, which relies on the fact that if there is a run, then there is also a periodic run. This method guesses a period and verifies that it does correspond to a run. To speed up this search, the period should be as short as possible. This motivates the notion of blocking automata.

Definition 2.2 (m-blocking) Let $\mathcal{A}=(Q, \Delta, I)$ be a looping automaton and $\longleftarrow$ a binary relation over $Q$ called the blocking relation. The automaton $\mathcal{A}$ is called $\longleftarrow$-invariant if $q \longleftarrow p$ and $\left(q_{0}, q_{1}, \ldots, q_{i-1}, q, q_{i+1}, \ldots, q_{k}\right) \in \Delta$ always imply $\left(q_{0}, q_{1}, \ldots, q_{i-1}, p, q_{i+1}, \ldots, q_{k}\right) \in \Delta$.

A $\longleftarrow$-invariant automaton is $m$-blocking for $m \in \mathbb{N}$ if every path $v_{1}, \ldots, v_{m}$ of length $m$ in a run $r$ of $\mathcal{A}$ contains two nodes $v_{i}$ and $v_{j}(i<j)$ such that $r\left(v_{j}\right) \longleftarrow r\left(v_{i}\right)$.

Every looping automaton is =-invariant and $m$-blocking for every $m>|Q|$. However, the main interest in blocking automata arises when one can find a smaller bound on $m$. Although this is not always possible, one can try to reduce this limit with the help of a so-called faithful family of functions.

Definition 2.3 (faithful) Let $\mathcal{A}=(Q, \Delta, I)$ be a looping automaton on $k$-ary trees. The family of functions $f_{q}: Q \rightarrow Q$ for $q \in Q$ is faithful w.r.t. $\mathcal{A}$ if for all $q, q_{0}, q_{1}, \ldots, q_{k} \in Q$, if $\left(q, q_{1}, \ldots, q_{k}\right) \in \Delta$, then $\left(q, f_{q}\left(q_{1}\right), \ldots, f_{q}\left(q_{k}\right)\right) \in \Delta$, and if $\left(q_{0}, q_{1}, \ldots, q_{k}\right) \in \Delta$, then $\left(f_{q}\left(q_{0}\right), f_{q}\left(q_{1}\right), \ldots, f_{q}\left(q_{k}\right)\right) \in \Delta$. The subautomaton $\mathcal{A}^{S}=\left(Q, \Delta^{S}, I\right)$ of $\mathcal{A}$ induced by this family has the transition relation

$\Delta^{S}=\left\{\left(q, f_{q}\left(q_{1}\right), \ldots, f_{q}\left(q_{k}\right)\right) \mid\left(q, q_{1}, \ldots, q_{k}\right) \in \Delta\right\}$.

Lemma 2.4 [2] Let $\mathcal{A}$ be a looping automaton and $\mathcal{A}^{S}$ its subautomaton induced by a faithful family of functions. Then $\mathcal{A}$ has a run iff $\mathcal{A}^{S}$ has a run.

The construction that we will present in Sect. 4 produces automata that are exponential in the size of the input. For such cases, it has been shown that if the automata are $m$-blocking for some $m$ bounded polynomially in the size of the input (that is, logarithmically in the size of the automaton), then the emptiness test requires only polynomial space.

Definition 2.5 (PSPACE on-the-fly construction) Assume that we have a set $\mathfrak{I}$ of inputs and a construction that yields, for every $\mathfrak{i} \in \mathfrak{I}$, an $m_{\mathfrak{i}}$-blocking automaton $\mathcal{A}_{\mathfrak{i}}=\left(Q_{\mathfrak{i}}, \Delta_{\mathfrak{i}}, I_{\mathfrak{i}}\right)$ working on $k_{i}$-ary trees. This construction is a PSPACE on-the-fly construction if there is a polynomial $P$ such that, for every input $i$ of size $n$

(i) $m_{\mathfrak{i}} \leq P(n)$ and $k_{\mathfrak{i}} \leq P(n)$,

(ii) every element of $Q_{\mathfrak{i}}$ has size bounded by $P(n)$, and

(iii) one can non-deterministically guess in time bounded by $P(n)$ an element of $I_{\mathfrak{i}}$, and, for a state $q \in Q_{\mathfrak{i}}$, a transition from $\Delta_{\mathfrak{i}}$ with first component $q$.

Theorem 2.6 [2] If the looping automata $\mathcal{A}_{\mathfrak{i}}$ are obtained from the inputs $\mathfrak{i} \in \mathfrak{I}$ by a PSPACE on-the-fly construction, then emptiness of $\mathcal{A}_{\mathfrak{i}}$ can be decided in PSPACE.

In Sect. 5, we will use this theorem to give PSPACE upper bounds for some reasoning problems. Example B.1 in the appendix presents a small looping automaton and a PSPACE on-the-fly construction.

\subsection{Residuated Lattices}

A lattice is an algebraic structure $(L, \vee, \wedge)$ over a carrier set $L$ with the two binary operations join $\vee$ and meet $\wedge$ that are idempotent, associative, and commutative and satisfy the absorption laws $\ell_{1} \vee\left(\ell_{1} \wedge \ell_{2}\right)=\ell_{1}=\ell_{1} \wedge\left(\ell_{1} \vee \ell_{2}\right)$ for all $\ell_{1}, \ell_{2} \in L$. These operations induce a partial order $\leq$ on $L$, defined by $\ell_{1} \leq \ell_{2}$ iff $\ell_{1} \wedge \ell_{2}=\ell_{1}$ for all $\ell_{1}, \ell_{2} \in L$. 


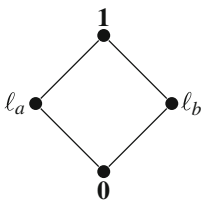

Fig. 2 The De Morgan lattice $L_{2}$ with $\sim \ell_{a}=\ell_{a}$ and $\sim \ell_{b}=\ell_{b}$

As usual, we write $\ell_{1}<\ell_{2}$ if $\ell_{1} \leq \ell_{2}$ and $\ell_{1} \neq \ell_{2}$. A subset $T \subseteq L$ is called an antichain (in $L$ ) if there are no two elements $\ell_{1}, \ell_{2} \in T$ with $\ell_{1}<\ell_{2}$.

The lattice $(L, \vee, \wedge)$ is called distributive if $\vee$ and $\wedge$ distribute over each other, finite if $L$ is finite, and bounded if it has a minimum and a maximum element, denoted as $\mathbf{0}$ and $\mathbf{1}$, respectively. It is complete if joins and meets of arbitrary subsets $T \subseteq L$, denoted by $\bigvee_{\ell \in T} \ell$ and $\bigwedge_{\ell \in T} \ell$ respectively, exist. As a simple consequence of these definitions, every finite lattice is also complete, and every complete lattice is bounded, with $\mathbf{0}=\bigwedge_{\ell \in L} \ell$ and $\mathbf{1}=\bigvee_{\ell \in L} \ell$. Whenever it is clear from the context, we will simply use the carrier set $L$ to represent the lattice $(L, \vee, \wedge)$.

A De Morgan lattice is a bounded distributive lattice $L$ extended with an involutive, anti-monotonic unary operation $\sim$, called (De Morgan) negation, satisfying the De Morgan laws $\sim\left(\ell_{1} \vee \ell_{2}\right)=\sim \ell_{1} \wedge \sim \ell_{2}$ and $\sim\left(\ell_{1} \wedge \ell_{2}\right)=\sim \ell_{1} \vee \sim \ell_{2}$ for all $\ell_{1}, \ell_{2} \in L$. Figure 2 shows a simple De Morgan lattice, where $\sim \ell_{a}=\ell_{a}, \sim \ell_{b}=\ell_{b}, \sim \mathbf{1}=\mathbf{0}$ and $\sim \mathbf{0}=\mathbf{1}$. This lattice was originally introduced in [5] to reason about incomplete and contradictory knowledge. The truth value $\ell_{a}$ indicates missing information, while $\ell_{b}$ labels a statement that is both true and false, e.g. according to different sources of information.

Given a lattice $L$, a $t$-norm is an associative and commutative binary operator on $L$ that is monotonic and has $\mathbf{1}$ as its unit. A residuated lattice is a lattice $L$ with a $t$-norm $\otimes$ and a binary operator $\Rightarrow$ (called residuum) such that for every three elements $\ell_{1}, \ell_{2}, \ell_{3} \in L$ we have

$\ell_{1} \otimes \ell_{2} \leq \ell_{3}$ iff $\ell_{2} \leq \ell_{1} \Rightarrow \ell_{3}$.

A simple consequence of this definition is that for all pairs of elements $\ell_{1}, \ell_{2} \in L$ we have

$-\mathbf{1} \Rightarrow \ell_{1}=\ell_{1}$, and

$-\ell_{1} \leq \ell_{2}$ iff $\ell_{1} \Rightarrow \ell_{2}=\mathbf{1}$.

In a complete lattice $L$, a $t$-norm $\otimes$ is called continuous if for all $\ell \in L$ and $T \subseteq L$ we have $\ell \otimes\left(\bigvee_{\ell^{\prime} \in T} \ell^{\prime}\right)=$ $\bigvee_{\ell^{\prime} \in T}\left(\ell \otimes \ell^{\prime}\right)$. In this case, $L$ is residuated with the unique residuum defined by

$\ell_{1} \Rightarrow \ell_{2}=\bigvee\left\{x \mid \ell_{1} \otimes x \leq \ell_{2}\right\}$.

For a residuated De Morgan lattice $L$, the $t$-conorm $\oplus$ is defined as $\ell_{1} \oplus \ell_{2}:=\sim\left(\sim \ell_{1} \otimes \sim \ell_{2}\right)$. It is easy to see that the meet operator $\ell_{1} \wedge \ell_{2}$ always defines a $t$-norm (called the Gödel $t$-norm), whose t-conorm is the join $\ell_{1} \vee \ell_{2}$. This $t$-norm is continuous if $L$ is completely distributive. Thus, if we extend the lattice $L_{2}$ from Fig. 2 with the Gödel $t$-norm, we obtain a residuated lattice, and e.g. $\ell_{a} \Rightarrow \ell_{b}=\ell_{b}$.

A prominent example of a residuated lattice is the real interval $[0,1]$ with the usual order and Gödel $t$-norm that is the basis of fuzzy set theory [33]. This theory defines fuzzy sets as a generalization of classical sets and uses the lattice operations to generalize the set operations such as intersection, union, and complement. Fuzzy set theory has also been extended to complete residuated De Morgan lattices [20,34], as we now briefly describe.

Given a residuated De Morgan lattice $L$, a fuzzy set is a function $A: D \rightarrow L$ that maps the elements of a domain $D$ to values in $L$. The value $A(d)$ for $d \in D$ specifies the degree to which $d$ belongs to $A$. The fuzzy intersection $C$ of two fuzzy sets $A, B$ over the domain $D$ is defined by

$C(d)=A(d) \otimes B(d)$

for all $d \in D$. Similarly, fuzzy union is defined using the t-conorm $\oplus$ and fuzzy complement using the De Morgan negation $\sim$.

Given two fuzzy sets $A, B$ over the domain $D$, the inclusion degree of $A$ in $B$ is

$\bigwedge_{d \in D} A(d) \Rightarrow B(d)$

This value is $\mathbf{1}$ iff $A(d) \leq B(d)$ holds for all $d \in D$. The idea is that, the higher the inclusion degree is, the closer $A$ is to being "properly included" in $B$.

A fuzzy binary relation is a fuzzy set $R: D \times D \rightarrow L$ on the domain $D \times D$. This function is called transitive if for all $d, e, f \in D$ we have $R(d, e) \otimes R(e, f) \leq R(d, f)$.

\section{The Fuzzy Description Logic $L-\mathcal{S H} \mathcal{I}$}

For the rest of this paper, $L$ denotes a complete residuated De Morgan lattice with the $t$-norm $\otimes$. The fuzzy DL $L-\mathcal{S H \mathcal { I }}$ is a generalization of the crisp DL $\mathcal{S H \mathcal { I }}$ that uses the elements of $L$ as truth values, instead of just the Boolean true and false. The syntax of $L-\mathcal{S H I}$ is the same as that of $\mathcal{S H I}$.

Definition 3.1 (syntax) Let $\mathrm{N}_{\mathrm{C}}$ and $\mathrm{N}_{\mathrm{R}}$ be two disjoint sets of concept names and role names, respectively, and the subset $\mathrm{N}_{\mathrm{R}}^{+} \subseteq \mathrm{N}_{\mathrm{R}}$ be the set of transitive role names. The set of (complex) roles is $\mathrm{N}_{\mathrm{R}} \cup\left\{r^{-} \mid r \in \mathrm{N}_{\mathrm{R}}\right\}$. The set of (complex) concepts is defined inductively as follows:

- every concept name $A \in \mathrm{N}_{\mathrm{C}}$ is a concept;

- if $C$ and $D$ are concepts and $s$ is a complex role, then the conjunction $C \sqcap D$, the disjunction $C \sqcup D$, the 
implication $C \rightarrow D$, the negation $\neg C$, the existential restriction $\exists s$. $C$, the value restriction $\forall s$. $C$, the top concept $\top$, and the bottom concept $\perp$ are also concepts.

The inverse of a complex role $s$ (denoted by $\bar{s}$ ) is $s^{-}$if $s \in \mathrm{N}_{\mathrm{R}}$ and $r$ if $s=r^{-}$. A role $s$ is transitive if either $s$ or $\bar{s}$ belongs to $\mathrm{N}_{\mathrm{R}}^{+}$.

The semantics of fuzzy description logics is based on interpretation functions that map every concept $C$ to a fuzzy set over $L$ that specifies the membership degree of every domain element to the concept $C$.

Definition 3.2 (semantics) An interpretation is a pair of the form $\mathcal{I}=\left(\Delta^{\mathcal{I}},{ }^{\mathcal{I}}\right)$ where $\Delta^{\mathcal{I}}$ is a non-empty set, called the domain of $\mathcal{I}$, and ${ }^{\mathcal{I}}$ is a function that assigns to every concept name $A$ a fuzzy set $A^{\mathcal{I}}: \Delta^{\mathcal{I}} \rightarrow L$ and to every role name $r$ a fuzzy binary relation $r^{\mathcal{I}}: \Delta^{\mathcal{I}} \times \Delta^{\mathcal{I}} \rightarrow L$ that is transitive whenever $r \in \mathrm{N}_{\mathrm{R}}^{+}$.

The function ${ }^{\mathcal{I}}$ is extended to complex roles and concepts as follows for every $x, y \in \Delta^{\mathcal{I}}$ :

- $\left(r^{-}\right)^{\mathcal{I}}(x, y)=r^{\mathcal{I}}(y, x)$,

$-\top^{\mathcal{I}}(x)=\mathbf{1}$,

$-\perp^{\mathcal{I}}(x)=\mathbf{0}$,

$-(C \sqcap D)^{\mathcal{I}}(x)=C^{\mathcal{I}}(x) \otimes D^{\mathcal{I}}(x)$,

- $(C \sqcup D)^{\mathcal{I}}(x)=C^{\mathcal{I}}(x) \oplus D^{\mathcal{I}}(x)$,

- $(C \rightarrow D)^{\mathcal{I}}(x)=C^{\mathcal{I}}(x) \Rightarrow D^{\mathcal{I}}(x)$,

- $(\neg C)^{\mathcal{I}}(x)=\sim C^{\mathcal{I}}(x)$,

- $(\exists s . C)^{\mathcal{I}}(x)=\bigvee_{z \in \Delta^{\mathcal{I}}}\left(s^{\mathcal{I}}(x, z) \otimes C^{\mathcal{I}}(z)\right)$, and

- $(\forall s . C)^{\mathcal{I}}(x)=\bigwedge_{z \in \Delta^{\mathcal{I}}}\left(s^{\mathcal{I}}(x, z) \Rightarrow C^{\mathcal{I}}(z)\right)$.

We emphasize that $\mathcal{S H \mathcal { I }}$ is a special case of $L-\mathcal{S H} \mathcal{H}$, where concepts are interpreted by classical sets. That is, the underlying lattice contains only the elements $\mathbf{0}$ and $\mathbf{1}$, which are interpreted as false and true, respectively, and the $t$-norm, the $t$-conorm, and the residuum are conjunction, disjunction, and implication, respectively.

For an arbitrary lattice $L$, existential and value restrictions need not be dual to each other, i.e. $(\neg \exists s . C)^{\mathcal{I}}$ might be different from $(\forall s . \neg C)^{\mathcal{I}}$, as illustrated by the following example.

Example 3.3 Consider the simple lattice $L_{2}$ from Fig. 2 with the Gödel $t$-norm and $\mathcal{I}$ be an interpretation over the domain $\Delta^{\mathcal{I}}=\{x\}$ with $A^{\mathcal{I}}(x)=\ell_{b}$ and $s^{\mathcal{I}}(x, x)=\ell_{a}$. Then $(\exists s . A)^{\mathcal{I}}(x)=\ell_{a} \wedge \ell_{b}=\mathbf{0}$, and thus $(\neg \exists s . A)^{\mathcal{I}}(x)=\mathbf{1}$, but $(\forall s . \neg A)^{\mathcal{I}}(x)=\ell_{a} \Rightarrow \sim \ell_{b}=\ell_{b} \neq \mathbf{1}$.

In DLs, the knowledge of a domain is represented by a set of axioms that restrict the set of interpretations that can be considered for reasoning. In the presence of fuzzy semantics, these axioms can also have an associated value from the lattice $L$, which intuitively expresses the degree to which they must be satisfied.
Definition 3.4 (axioms) Terminological axioms are concept definitions of the form $\langle A \doteq C, \ell\rangle$ or general concept inclusions (GCIs) $\langle C \sqsubseteq D, \ell\rangle$, where $A \in \mathrm{N}_{\mathrm{C}}, C, D$ are $L-\mathcal{S H} \mathcal{I}$ concepts, and $\ell \in L$. A general TBox is a finite set of GCIs. An acyclic TBox is a finite set of concept definitions such that every concept name occurs at most once as the left-hand side of an axiom, and there is no cyclic dependency between defined concept names, where a concept name $A$ depends on another concept name $B$ if $B$ occurs in a definition of the form $\langle A \doteq C, \ell\rangle$ in the TBox. A TBox is either a general TBox or an acyclic TBox.

Role inclusions are of the form $s \sqsubseteq s^{\prime}$, where $s$ and $s^{\prime}$ are complex roles. An RBox is a finite set of role inclusions. An ontology $(\mathcal{T}, \mathcal{R})$ consists of a TBox $\mathcal{T}$ and an $\operatorname{RBox} \mathcal{R}$.

An interpretation $\mathcal{I}$ satisfies the GCI $\langle C \sqsubseteq D, \ell\rangle$ if for every $x \in \Delta^{\mathcal{I}}, C^{\mathcal{I}}(x) \Rightarrow D^{\mathcal{I}}(x) \geq \ell$ holds. It satisfies the concept definition $\langle A \doteq C, \ell\rangle$ if for every $x \in \Delta^{\mathcal{I}}$, it holds that $\left(A^{\mathcal{I}}(x) \Rightarrow C^{\mathcal{I}}(x)\right) \otimes\left(C^{\mathcal{I}}(x) \Rightarrow A^{\mathcal{I}}(x)\right) \geq \ell$. It satisfies the role inclusion $s \sqsubseteq s^{\prime}$ if $s^{\mathcal{I}}(x, y) \leq s^{\prime \mathcal{I}}(x, y)$ for every $x, y \in \Delta^{\mathcal{I}}$. $\mathcal{I}$ is a model of the ontology $(\mathcal{T}, \mathcal{R})$ if it satisfies all axioms in $\mathcal{T}$ and $\mathcal{R}$.

Notice that general TBoxes are indeed more general than acyclic TBoxes: the concept definition $\langle A \doteq C, \ell\rangle$ can be expressed by the GCI $\langle\top \sqsubseteq(A \rightarrow C) \sqcap(C \rightarrow A), \ell\rangle$.

If $\mathcal{T}$ is an acyclic TBox, then all concept names occurring on the left-hand side of some axiom of $\mathcal{T}$ are called defined, all others are called primitive. If $\mathcal{T}$ is a general TBox, then all concept names appearing in it are primitive.

Given an RBox $\mathcal{R}$, the role hierarchy $\subseteq_{\mathcal{R}}$ over the set of complex roles is the reflexive and transitive closure of the relation

$\left\{\left(s, s^{\prime}\right) \mid s \sqsubseteq s^{\prime} \in \mathcal{R}\right.$ or $\left.\bar{s} \sqsubseteq \overline{s^{\prime}} \in \mathcal{R}\right\}$.

Using a reachability algorithm, the role hierarchy can be computed in polynomial time in the size of $\mathcal{R}$. An RBox $\mathcal{R}$ is acyclic if it induces no cycles of the form $s \sqsubseteq \mathcal{R} s^{\prime}, s^{\prime} \sqsubseteq \mathcal{R} s$ for two complex roles $s \neq s^{\prime}$.

We also consider several sublogics of $L-\mathcal{S H} \mathcal{I}$. If transitive roles are not allowed in $L-\mathcal{S H} \mathcal{H}$, i.e. $\mathrm{N}_{\mathrm{R}}^{+}=\emptyset$, then we obtain the fuzzy DL $L-\mathcal{A} \mathcal{L C H} \mathcal{H}$. Disallowing role inclusions, i.e. setting $\mathcal{R}=\emptyset$, yields the logic $L-\mathcal{S} \mathcal{I}$. Leaving out both role inclusions and transitive roles, produces the logic $L-\mathcal{A L C I}$. Disallowing the inverse role constructor from this last logic leaves $L-\mathcal{A L C}$. Other logics like $L-\mathcal{A} \mathcal{L C H}, L-\mathcal{S}$, and $L-\mathcal{S H}$ (see Fig. 1) can also be produced by disallowing some of the constructors or axioms used in $L-\mathcal{S H I}$. The description logic $L-S \mathcal{I}_{\mathrm{c}}$ further restricts the semantics of $L-\mathcal{S I}$ by forcing roles to be crisp, i.e. $r^{\mathcal{I}}(x, y) \in\{\mathbf{0}, \mathbf{1}\}$ for all interpretations $\mathcal{I}, r \in \mathrm{N}_{\mathrm{R}}$, and $x, y \in \Delta^{\mathcal{I}}$.

The standard reasoning problems for crisp DLs can be generalized to also take into account the membership degrees 
provided by the lattice-based semantics. We will focus on the problem of local consistency, which generalizes classical satisfiability and is equivalent to satisfiability in the crisp case. It can be used to also decide strong $\ell$-satisfiability and $\ell$-subsumption w.r.t. finite lattices $[12,13]$.

Definition 3.5 (decision problems) Let $C$ and $D$ be two concepts, $\mathcal{O}$ an ontology, and $\ell \in L$. We say that $C$ is strongly $\ell$-satisfiable w.r.t. $\mathcal{O}$ if there is a model $\mathcal{I}$ of $\mathcal{O}$ and an $x \in \Delta^{\mathcal{I}}$ such that $C^{\mathcal{I}}(x) \geq \ell$. $C$ is $\ell$-subsumed by $D$ w.r.t. $\mathcal{O}$ if every model $\mathcal{I}$ of $\mathcal{O}$ satisfies $\langle C \sqsubseteq D, \ell\rangle$.

A local ABox $\mathbf{A}$ is a finite set of pairs $(C, \ell)$, where $C$ is a concept, and $\ell \in L$. Such a local ABox $\mathbf{A}$ is locally consistent w.r.t. $\mathcal{O}$ if there is a model $\mathcal{I}$ of $\mathcal{O}$ and $x \in \Delta^{\mathcal{I}}$ such that $C^{\mathcal{I}}(x)=\ell$ for all $(C, \ell) \in \mathbf{A}$.

A local ABox is a special form of a finite set of assertions, called ABox, which is often used in DL reasoning. ABoxes are more expressive than local ABoxes, as they can express restrictions on several domain elements and restrict the role connections between these individuals. Moreover, inequality restrictions of the form $C^{\mathcal{I}}(x) \geq \ell$ are usually allowed in ABoxes [9,30]. From this point of view, a local ABox can be seen as an $\mathrm{ABox}$ that is restricted to equality assertions on one individual.

For infinite lattices, local consistency has been shown to be undecidable even in the logic $L-\mathcal{A L C}$ [15]. We therefore restrict our attention to finite residuated De Morgan lattices.

Notice that in the case of a finite lattice $L$, strong satisfiability and (non-)subsumption of concepts can be reduced to local consistency as follows. A concept $C$ is strongly $\ell$-satisfiable iff there is an $\ell^{\prime} \geq \ell$ such that $\left\{\left(C, \ell^{\prime}\right)\right\}$ is locally consistent, and $C$ is not $\ell$-subsumed by $D$ iff there is an $\ell^{\prime} \geq \ell$ such that $\left\{\left(C \rightarrow D, \ell^{\prime}\right)\right\}$ is locally consistent. In the crisp case, local consistency is in fact equivalent to satisfiability: the local ABox $\mathbf{A}$ is locally consistent iff the concept

$$
\prod_{(C, \mathbf{1}) \in \mathbf{A}} C \sqcap \prod_{(C, \mathbf{0}) \in \mathbf{A}} \neg C
$$

is satisfiable.

Recall that the semantics of the quantifiers require the computation of a supremum or infimum of the membership degrees of a possibly infinite subset of the domain. To obtain effective decision procedures, it is customary to restrict reasoning to witnessed models [23].

Definition 3.6 (witnessed interpretation) Let $n \in \mathbb{N}$. An interpretation $\mathcal{I}$ is called $n$-witnessed if for every $x \in \Delta^{\mathcal{I}}$ and every concept $C$ and role $r$ there are elements $x_{1}, \ldots, x_{n} \in \Delta^{\mathcal{I}}$ and $y_{1}, \ldots, y_{n} \in \Delta^{\mathcal{I}}$ such that

$$
\begin{aligned}
& (\exists r . C)^{\mathcal{I}}(x)=\bigvee_{i=1}^{n} r^{\mathcal{I}}\left(x, x_{i}\right) \otimes C^{\mathcal{I}}\left(x_{i}\right), \text { and } \\
& (\forall r . C)^{\mathcal{I}}(x)=\bigwedge_{i=1}^{n} r^{\mathcal{I}}\left(x, y_{i}\right) \Rightarrow C^{\mathcal{I}}\left(y_{i}\right) .
\end{aligned}
$$

In particular, if $n=1$, then the suprema and infima from the semantics of $\exists r . C$ and $\forall r . C$ become maxima and minima, respectively. In this case, we say that $\mathcal{I}$ is witnessed.

We will show in the following sections that most of the fuzzy description logics introduced above exhibit the same complexity upper bounds for deciding local consistency as their crisp counterparts, i.e. the lattice-based semantics does not increase the complexity of reasoning in these logics.

\section{Deciding Local Consistency}

Recall that we deal only with finite residuated De Morgan lattices. For the special case of finite total orders, a popular approach is to reduce the fuzzy ontology to a crisp one $[7,10,11,30]$, and a similar method would work also for arbitrary finite lattices. This approach allows to employ existing optimized reasoners for the actual reasoning. The main idea is to translate every concept name $A$ into finitely many crisp concept names $A_{\geq \ell}$, one for each membership degree $\ell$, with the intention that $A_{\geq \ell}$ collects all those individuals that belong to $A$ with a membership degree $\geq \ell$. Then, for every concept name $A$ and every pair $\left(\ell_{1}, \ell_{2}\right)$ of lattice elements such that $\ell_{2}$ is a minimal element above $\ell_{1}$, one has to introduce a GCI $A_{\geq \ell_{2}} \sqsubseteq A_{\geq \ell_{1}}$ to express the lattice structure. A similar translation is done for role names and all axioms are then recursively translated into crisp axioms that use only the introduced crisp concept and role names. The resulting crisp ontology is consistent in the classical sense iff the original fuzzy ontology is consistent.

However, in general, such a translation yields an inefficient reasoning procedure. Depending on the $t$-norm chosen, there might be $|L|^{2}$ different combinations of elements of $L$ whose $t$-norm is a given $\ell \in L$, and similarly for all the other constructors. All these possibilities must be expressed in the translation, which then produces an ontology of size in the order of $|L|^{2}|\mathcal{O}|$. Since the reasoning algorithm for crisp DLs is exponential on the size of the input ontology, this yields a runtime exponential in $|L|^{2}|\mathcal{O}|{ }^{6}$ As we will see, our automata-based approach allows us to reduce this bound to be exponential in $\log (|L|)|\mathcal{O}|$. Moreover, even if the original TBox is acyclic, the translation produces a general crisp TBox for which reasoning remains exponential. This is suboptimal since, as we show in Sect. 5, reasoning w.r.t. acyclic TBoxes is in PSPACE.

We now show that deciding local consistency in $L-\mathcal{S H} \mathcal{I}$ is EXPTIME-complete. This matches the complexity of crisp $\mathcal{S H I}$ [31]. We assume for now that the lattice $L$ is fixed, and thus do not consider the size of $L$ in our complexity

\footnotetext{
6 For special lattices, in particular total orders with the so-called Zadeh semantics, this blowup can be avoided [30].
} 
analysis. This is not a strong assumption, since usually the truth degrees are decided and fixed for a given application. In Sect. 6, we will comment briefly on the influence of $L$ on the complexity of our algorithms.

Without loss of generality, we consider reasoning w.r.t. $n$-witnessed models: since $L$ is finite, $L-\mathcal{S H} \mathcal{I}$ always has the $|L|$-witnessed model property. It is possible to obtain a smaller bound on the number of required witnesses, depending on the structure of the lattice.

Lemma 4.1 If the maximal cardinality of an antichain of $L$ is $n$, then every interpretation in $L-\mathcal{S H I}$ is $n$-witnessed.

Proof Let $m$ be the smallest natural number such that there exist $y_{1}, \ldots, y_{m} \in \Delta^{\mathcal{I}}$ with

$(\exists r . C)^{\mathcal{I}}(x)=\bigvee_{i=1}^{m}\left(r^{\mathcal{I}}\left(x, y_{i}\right) \otimes C^{\mathcal{I}}\left(y_{i}\right)\right)$.

From the minimality of $m$, we know that this supremum cannot be reached using only $m-1$ different values of the form $r^{\mathcal{I}}\left(x, y_{i}\right) \otimes C^{\mathcal{I}}\left(y_{i}\right)$. Thus, removing any element from $\left\{y_{1}, \ldots, y_{m}\right\}$ decreases this supremum. This can only be the case if the values $r^{\mathcal{I}}\left(x, y_{i}\right) \otimes C^{\mathcal{I}}\left(y_{i}\right)$ are all incomparable, i.e. for every $i \neq j$ it holds that

$r^{\mathcal{I}}\left(x, y_{i}\right) \otimes C^{\mathcal{I}}\left(y_{i}\right) \not \leq r^{\mathcal{I}}\left(x, y_{j}\right) \otimes C^{\mathcal{I}}\left(y_{j}\right) ;$

otherwise, removing $y_{i}$ would yield the same supremum. This means that the set $\left\{r^{\mathcal{I}}\left(x, y_{i}\right) \otimes C^{\mathcal{I}}\left(y_{i}\right) \mid 1 \leq i \leq m\right\}$ forms an antichain of cardinality $m$. By assumption, $m \leq n$. This shows that $\mathcal{I}$ is $n$-witnessed. ${ }^{7}$

For example, if $L$ is a total order, then $L-\mathcal{S H} \mathcal{I}$ has the witnessed model property. To simplify the description of the algorithm, in the following we consider only the special case $n=1$, i.e. we restrict reasoning to witnessed models only. As shown in Example 4.2 below, in general $L-\mathcal{A L C}$ does not have the witnessed model property; however, the algorithm and the proofs of correctness can easily be adapted for any other $n \in \mathbb{N}$ (see Sect. 6).

Example 4.2 Consider the lattice $L_{2}$ from Fig. 2 with the Gödel $t$-norm, and the TBox

$\mathcal{T}=\{\langle\top \sqsubseteq(A \sqcap \neg A) \sqcup(B \sqcap \neg B), \mathbf{1}\rangle\}$.

The local ABox $\mathbf{A}=\{(\exists r . A, \mathbf{1})\}$ is locally consistent w.r.t. $\mathcal{T}$ since the interpretation $\mathcal{I}_{0}=\left(\left\{x, y_{1}, y_{2}\right\},{ }^{\mathcal{I}_{0}}\right)$ with

$$
\begin{gathered}
r^{\mathcal{I}_{0}}\left(x, y_{1}\right)=r^{\mathcal{I}_{0}}\left(x, y_{2}\right)=\mathbf{1}, \\
A^{\mathcal{I}_{0}}(x)=A^{\mathcal{I}_{0}}\left(y_{1}\right)=B^{\mathcal{I}_{0}}\left(y_{2}\right)=\ell_{a}, \text { and } \\
B^{\mathcal{I}_{0}}(x)=B^{\mathcal{I}_{0}}\left(y_{1}\right)=A^{\mathcal{I}_{0}}\left(y_{2}\right)=\ell_{b}
\end{gathered}
$$

7 It is easy to find the remaining $n-m$ witnesses. For each $y \in \Delta^{\mathcal{I}}$ we have $r^{\mathcal{I}}(x, y) \otimes C^{\mathcal{I}}(y) \leq(\exists r . C)^{\mathcal{I}}(x)$ and thus, any choice of additional elements of the domain would suffice. is a model of $\mathcal{T}$ and $(\exists r . A)^{\mathcal{I}_{0}}(x)=A^{\mathcal{I}_{0}}\left(y_{1}\right) \vee A^{\mathcal{I}_{0}}\left(y_{2}\right)=\mathbf{1}$. However, $\mathbf{A}$ is not locally consistent in any witnessed model of $\mathcal{T}$ : since $\ell \wedge \sim \ell \neq \mathbf{1}$ for every $\ell \in L_{2}$, the only axiom in $\mathcal{T}$ can only be satisfied if for every $y \in \Delta^{\mathcal{I}}$, it holds that $\left\{A^{\mathcal{I}}(y), B^{\mathcal{I}}(y)\right\}=\left\{\ell_{a}, \ell_{b}\right\}$, and in particular if $A^{\mathcal{I}}(y)<\mathbf{1}$. Thus, there can be no unique witness for the concept $\exists r . A$ having degree $\mathbf{1}$.

Even if $L-S \mathcal{H I}$ does not have the witnessed model property, one might want to restrict reasoning to witnessed models to avoid unintuitive consequences. For example, an interpretation might satisfy the axiom $\langle\top \sqsubseteq \exists r . \top, \mathbf{1}\rangle$, but it is not guaranteed that every domain element has a "true" $r$-successor, i.e. one with degree $\mathbf{1}$. Restricting reasoning to witnessed models is a standard assumption in fuzzy description logics (see for example [4,6,23]).

Our algorithm for deciding local consistency exploits the fact that an ontology $\mathcal{O}$ has a (witnessed) model iff it has a well-structured tree-shaped model, called a Hintikka tree. Intuitively, Hintikka trees are abstract representations of models that explicitly express the membership value of all "relevant" concepts. We will construct automata that have exactly these Hintikka trees as their runs, and use the initial states to verify that an element in the model verifies the local consistency condition. Reasoning is hence reduced to testing emptiness of these automata.

The set $\operatorname{sub}_{\mathcal{R}}(C)$ of subconcepts of an $L-\mathcal{S H} \mathcal{I}$ concept $C$ w.r.t. an RBox $\mathcal{R}$ is defined inductively as follows:

$$
\begin{aligned}
& -\operatorname{sub}_{\mathcal{R}}(A)=\{A\} \text { if } A \text { is a concept name, } \top, \text { or } \perp, \\
& -\operatorname{sub}_{\mathcal{R}}(C)=\{C\} \cup \operatorname{sub}_{\mathcal{R}}(D) \cup \operatorname{sub}_{\mathcal{R}}(E) \\
& \quad \text { if } C \text { is of the form } D \sqcap E, D \sqcup E, \text { or } D \rightarrow E, \\
& -\operatorname{sub}_{\mathcal{R}}(\neg C)=\{\neg C\} \cup \operatorname{sub}_{\mathcal{R}}(C), \\
& -\operatorname{sub}_{\mathcal{R}}(\exists s . C)=\{\exists s . C\} \cup \operatorname{sub}_{\mathcal{R}}(C) \\
& \cup\left\{\exists s^{\prime} . C \mid s^{\prime} \sqsubseteq \mathcal{R} s, s^{\prime} \text { is transitive }\right\} \\
& -\operatorname{sub}_{\mathcal{R}}(\forall s . C)=\{\forall s . C\} \cup \operatorname{sub}_{\mathcal{R}}(C) \\
& \cup\left\{\forall s^{\prime} . C \mid s^{\prime} \sqsubseteq \mathcal{R} s, s^{\prime} \text { is transitive }\right\}
\end{aligned}
$$

For a local ABox $\mathbf{A}$ and an ontology $\mathcal{O}=(\mathcal{T}, \mathcal{R})$, the set of all subconcepts of every concept appearing in $\mathbf{A}$ or $\mathcal{O}$ is

$$
\begin{aligned}
\operatorname{sub}(\mathbf{A}, \mathcal{O})= & \bigcup_{(C, \ell) \in \mathbf{A}} \operatorname{sub}_{\mathcal{R}}(C) \cup \\
& \bigcup_{\langle E \sqsubseteq F, \ell\rangle \in \mathcal{T}}\left(\operatorname{sub}_{\mathcal{R}}(E) \cup \operatorname{sub}_{\mathcal{R}}(F)\right) \cup \\
& \bigcup_{\langle A \doteq F, \ell\rangle \in \mathcal{T}}\left(\{A\} \cup \operatorname{sub}_{\mathcal{R}}(F)\right) .
\end{aligned}
$$

The nodes of Hintikka trees are labeled with so-called Hintikka functions, which are fuzzy sets over the domain $\operatorname{sub}(\mathbf{A}, \mathcal{O}) \cup\{\rho\}$, where $\rho$ is an arbitrary new element that will be used to express the degree with which the role relation to the parent node holds. 
Definition 4.3 (Hintikka function) A Hintikka function for $\mathbf{A}, \mathcal{O}$ is a partial function $H: \operatorname{sub}(\mathbf{A}, \mathcal{O}) \cup\{\rho\} \rightarrow L$ such that:

(i) $H(\rho)$ is defined,

(ii) if $H(\top)$ is defined, then $H(\top)=\mathbf{1}$,

(iii) if $H(\perp)$ is defined, then $H(\perp)=\mathbf{0}$,

(iv) if $H(D \sqcap E)$ is defined, then $H(D)$ and $H(E)$ are also defined and $H(D \sqcap E)=H(D) \otimes H(E)$,

(v) if $H(D \sqcup E)$ is defined, then $H(D)$ and $H(E)$ are also defined and $H(D \sqcup E)=H(D) \oplus H(E)$,

(vi) if $H(D \rightarrow E)$ is defined, then $H(D)$ and $H(E)$ are also defined and $H(D \rightarrow E)=H(D) \Rightarrow H(E)$,

(vii) if $H(\neg D)$ is defined, then $H(D)$ is also defined and $H(\neg D)=\sim H(D)$,

$H$ is compatible with the concept definition $\langle A \doteq E, \ell\rangle$ if, whenever $H(A)$ is defined, then $H(E)$ is defined and we have $(H(A) \Rightarrow H(E)) \otimes(H(E) \Rightarrow H(A)) \geq \ell$. It is compatible with the GCI $\langle E \sqsubseteq F, \ell\rangle$ if $H(E)$ and $H(F)$ are defined and $H(E) \Rightarrow H(F) \geq \ell$.

The set support $(H)$ contains all concepts $C$ in $\operatorname{sub}(\mathbf{A}, \mathcal{O})$ for which $H(C)$ is defined.

Hintikka trees have a fixed arity $k$ determined by the number of existential and value restrictions, i.e. concepts of the form $\exists s . F$ or $\forall s . F$, in $\operatorname{sub}(\mathbf{A}, \mathcal{O})$. Intuitively, each successor will act as the witness for one of these restrictions. As in Sect. 2.1, we define $K$ to be the index set $\{1, \ldots, k\}$ of all successors. Since we need to know which successor in the tree is the witness of which restriction, we fix an arbitrary bijection

$\varphi:\{E \mid E \in \operatorname{sub}(\mathbf{A}, \mathcal{O})$ is of the form $\exists s . F$ or $\forall s . F\} \rightarrow K$

Definition 4.4 (Hintikka condition) The tuple of Hintikka functions $\left(H_{0}, H_{1}, \ldots, H_{k}\right)$ satisfies the Hintikka condition if: for every existential restriction $\exists s . G \in \operatorname{sub}(\mathbf{A}, \mathcal{O})$, the following hold:

a) If $\exists s . G \in \operatorname{support}\left(H_{0}\right)$, then

- $G \in \operatorname{support}\left(H_{\varphi(\exists s . G)}\right)$ and

- $H_{0}(\exists s . G)=H_{\varphi(\exists s . G)}(\rho) \otimes H_{\varphi(\exists s . G)}(G)$.

b) If $\exists s . G \in \operatorname{support}\left(H_{0}\right)$, then for every $E \in \operatorname{sub}(\mathbf{A}, \mathcal{O})$ of the form $\exists s^{\prime} . F$ or $\forall s^{\prime} . F$ with $s^{\prime} \sqsubseteq \mathcal{R} s$,

- $G \in \operatorname{support}\left(H_{\varphi(E)}\right)$ and

- $H_{0}(\exists s . G) \geq H_{\varphi(E)}(\rho) \otimes H_{\varphi(E)}(G)$.

Moreover, for all transitive roles $r$ with $s^{\prime} \sqsubseteq \mathcal{R} r \sqsubseteq \mathcal{R} s$,

- $\exists r . G \in \operatorname{support}\left(H_{\varphi(E)}\right)$ and

- $H_{0}(\exists s . G) \geq H_{\varphi(E)}(\rho) \otimes H_{\varphi(E)}(\exists r . G)$. c) For every $E \in \operatorname{sub}(\mathbf{A}, \mathcal{O})$ of the form $\exists s^{\prime} . F$ or $\forall s^{\prime} . F$ with $s^{\prime} \sqsubseteq \mathcal{R} \bar{s}$ and $\exists s . G \in \operatorname{support}\left(H_{\varphi(E)}\right)$,

- $G \in \operatorname{support}\left(H_{0}\right)$ and

- $H_{\varphi(E)}(\exists s . G) \geq H_{\varphi(E)}(\rho) \otimes H_{0}(G)$.

Moreover, for all transitive roles $r$ with $s^{\prime} \sqsubseteq \mathcal{R} r \sqsubseteq \mathcal{R} \bar{s}$,

- $\exists r . G \in \operatorname{support}\left(H_{0}\right)$ and

- $H_{\varphi(E)}(\exists s . G) \geq H_{\varphi(E)}(\rho) \otimes H_{0}(\exists r . G)$, and

for every value restriction $\forall s . G \in \operatorname{sub}(\mathbf{A}, \mathcal{O})$, the following hold:

a') If $\forall s . G \in \operatorname{support}\left(H_{0}\right)$, then

- $G \in \operatorname{support}\left(H_{\varphi(\forall s . G)}\right)$ and

- $H_{0}(\forall s . G)=H_{\varphi(\forall s . G)}(\rho) \Rightarrow H_{\varphi(\forall s . G)}(G)$.

b') If $\forall s . G \in \operatorname{support}\left(H_{0}\right)$, then for every $E \in \operatorname{sub}(\mathbf{A}, \mathcal{O})$ of the form $\exists s^{\prime} . F$ or $\forall s^{\prime} . F$ with $s^{\prime} \sqsubseteq \mathcal{R} s$,

- $G \in \operatorname{support}\left(H_{\varphi(E)}\right)$ and

- $H_{0}(\forall s . G) \leq H_{\varphi(E)}(\rho) \Rightarrow H_{\varphi(E)}(G)$.

Moreover, for all transitive roles $r$ with $s^{\prime} \sqsubseteq \mathcal{R} r \sqsubseteq \mathcal{R} s$,

- $\forall r . G \in \operatorname{support}\left(H_{\varphi(E)}\right)$ and

- $H_{0}(\forall s . G) \leq H_{\varphi(E)}(\rho) \Rightarrow H_{\varphi(E)}(\forall r . G)$.

c') For every $E \in \operatorname{sub}(\mathbf{A}, \mathcal{O})$ of the form $\exists s^{\prime} . F$ or $\forall s^{\prime} . F$ with $s^{\prime} \sqsubseteq \mathcal{R} \bar{s}$ and $\forall s . G \in \operatorname{support}\left(H_{\varphi(E)}\right)$,

- $G \in \operatorname{support}\left(H_{0}\right)$ and

- $H_{\varphi(E)}(\forall s . G) \leq H_{\varphi(E)}(\rho) \Rightarrow H_{0}(G)$.

Moreover, for all transitive roles $r$ with $s^{\prime} \sqsubseteq \mathcal{R} r \sqsubseteq \mathcal{R} \bar{s}$,

- $\forall r . G \in \operatorname{support}\left(H_{0}\right)$ and

- $H_{\varphi(E)}(\forall s . G) \leq H_{\varphi(E)}(\rho) \Rightarrow H_{0}(\forall r . G)$.

The tuple is compatible with the axiom $t$ if the Hintikka functions $H_{0}, \ldots, H_{k}$ are compatible with $t$.

We briefly explain the intuition behind this definition. Condition a) makes sure that an existential restriction $\exists s . G$ is witnessed by its designated successor $\varphi(\exists s . G)$. Condition b) ensures that the degree of the existential restriction is indeed the supremum of the degrees of all $s$-successors. Furthermore, for all transitive subroles $r$ of $s$ the restriction $\exists r . G$ has to be propagated since by transitivity every $r$-successor of this $r$-successor must also be an $r$-successor. Finally, Condition c) deals with the consequences of the restriction $\exists s . G$ along inverse role connections. Conditions a') to c') state the dual notions for value restrictions. Notice that if it holds that $H_{\varphi(E)}(\rho)=\mathbf{0}$, then the inequalities from Conditions b), c), b'), and c') are trivially satisfied.

A Hintikka tree for $\mathbf{A}, \mathcal{O}$ is an infinite $k$-ary tree $\mathbf{T}$ labeled with Hintikka functions for $\mathbf{A}, \mathcal{O}$ such that $\mathbf{T}(\varepsilon)(C)$ is defined 
for all $(C, \ell) \in \mathbf{A}$ and for every node $u \in K^{*}$, the tuple $(\mathbf{T}(u), \mathbf{T}(u 1), \ldots, \mathbf{T}(u k))$ satisfies the Hintikka condition and is compatible with all axioms in $\mathcal{O}$. Compatibility ensures that all axioms are satisfied at any node of the Hintikka tree, while the Hintikka condition makes sure that the tree is in fact a witnessed model.

The proof of the following theorem uses arguments that generalize those used in [2] for crisp $\mathcal{S I}$. The Hintikka condition in [2] is much simpler since Hintikka functions are only sets of subconcepts, no successors witnessing the value restrictions are needed, and there is no role hierarchy. The full proof of this theorem appears in the appendix.

Theorem 4.5 Let $\mathbf{A}$ be a local $A B o x$ and $\mathcal{O}$ an ontology. A is locally consistent w.r.t. $\mathcal{O}$ iff there is a Hintikka tree $\mathbf{T}$ for $\mathbf{A}, \mathcal{O}$ with $\mathbf{T}(\varepsilon)(C)=\ell$ for all $(C, \ell) \in \mathbf{A}$.

Thus, local consistency of $L-\mathcal{S H I}$ concepts can be reduced to deciding the existence of a Hintikka tree with additional restrictions in the root. By building looping automata whose runs correspond exactly to those Hintikka trees, we reduce reasoning in $L-\mathcal{S H} \mathcal{I}$ to the emptiness problem of these automata.

In Sect. 5.2, we will additionally need to know the index of each node relative to its siblings in the tree. We thus use as states of the automaton pairs of the form $(H, i)$ where $H$ is a compatible Hintikka function and $i \in K$.

Definition 4.6 (Hintikka automaton) Let $\mathcal{O}$ be an ontology and $\mathbf{A}$ a local ABox. The Hintikka automaton for $\mathbf{A}, \mathcal{O}$ is $\mathcal{A}_{\mathbf{A}, \mathcal{O}}=(Q, I, \Delta)$, where

- $Q$ is the set of all pairs $(H, i)$ of compatible Hintikka functions for $\mathbf{A}, \mathcal{O}$ and indices $i \in K$,

- $I$ is the set of all pairs $(H, i) \in Q$ with $H(C)=\ell$ for every $(C, \ell) \in \mathbf{A}$, and

- $\Delta$ is the set of all tuples $\left(\left(H_{0}, i_{0}\right),\left(H_{1}, 1\right), \ldots,\left(H_{k}, k\right)\right)$ such that $\left(H_{0}, \ldots, H_{k}\right)$ satisfies the Hintikka condition.

The first components of the runs of $\mathcal{A}_{\mathbf{A}, \mathcal{O}}$ form exactly the Hintikka trees $\mathbf{T}$ with $\mathbf{T}(\varepsilon)(C)=\ell$ for all $(C, \ell) \in \mathbf{A}$. The second component simply stores the index of the existential or universal restriction for which a node acts as a witness for its parent, but does not influence the transition relation. Thus, $\mathbf{A}$ is locally consistent w.r.t. $\mathcal{O}$ iff $\mathcal{A}_{\mathbf{A}, \mathcal{O}}$ is not empty. In Example B.2, we describe a simple local consistency problem and the resulting Hintikka automaton.

Recall that $k$ is bounded by the number $|\operatorname{sub}(\mathbf{A}, \mathcal{O})|$ of subconcepts in $\mathbf{A}$ and $\mathcal{O}$, which is polynomial in the size of $\mathbf{A}$ and $\mathcal{O}$. Since there are at most $(|L|+1)^{|\operatorname{sub}(\mathbf{A}, \mathcal{O})|+1}$ Hintikka functions, the size of the automaton $\mathcal{A}_{\mathbf{A}, \mathcal{O}}$ is therefore exponential in the input $\mathbf{A}, \mathcal{O}{ }^{8}$ Hence, local consistency in

\footnotetext{
8 Recall that we consider the lattice $L$ to be fixed, and thus it is not part of the input.
}

$L-\mathcal{S H I}$ can be decided in exponential time. w.r.t. general TBoxes is already EXPTIME-hard for crisp $\mathcal{A L C}$ [28].

Theorem 4.7 In all logics between $L-\mathcal{A L C}$ and $L-\mathcal{S H I}$, deciding local consistency, strong satisfiability, and subsumption w.r.t. general TBoxes is EXPTIME-complete.

This shows that reasoning w.r.t. general TBoxes is not harder than in crisp description logics and extends our previous result for $L-\mathcal{A L C I}$ [13].

Recall that concept satisfiability is EXPTIME-complete for crisp $\mathcal{S H}$, even if the TBox is empty [25]. It thus follows that local consistency, and hence strong satisfiability and subsumption, is EXPTIME-complete for $L-\mathcal{S H} \mathcal{I}$ w.r.t. acyclic or empty TBoxes. In the following section we show that for the sublogics $L-\mathcal{S I}_{\mathrm{C}}$ and $L-\mathcal{A} \mathcal{L C H} \mathcal{H}$, the restriction to acyclic TBoxes reduces the complexity of reasoning.

\section{PSPACE Results for Acyclic TBoxes}

When restricted to acyclic TBoxes, reasoning in crisp $\mathcal{S I}$ becomes PSPACE-complete $[2,25]$. This implies that local consistency in $L-\mathcal{S I}$ w.r.t. acyclic TBoxes is PSPACE-hard, leaving a gap to the EXPTIME upper bound shown in the previous section.

We can close this gap if we restrict to $L-\mathcal{S} \mathcal{I}_{\text {c }}$, i.e. local consistency is then decidable in PSPACE. We show the same for $L-\mathcal{A L C H} \mathcal{I}$ w.r.t. acyclic TBoxes, which yields a new result about the complexity of reasoning in the crisp description $\operatorname{logic} \mathcal{A L C H \mathcal { I }}$

In the following, let $\mathcal{O}=(\mathcal{T}, \mathcal{R})$ be an ontology where $\mathcal{T}$ is an acyclic TBox. Our goal is to obtain PSPACE decision procedures by modifying the construction of the Hintikka automata into PSPACE on-the-fly constructions. Notice that $\mathcal{A}_{\mathrm{A}, \mathcal{O}}$ satisfies all but one of the conditions of Definition 2.5:

(i) the arity $k$ of the automata is given by the number of existential and value restrictions in $\operatorname{sub}(\mathbf{A}, \mathcal{O})$,

(ii) every Hintikka function (and hence every state of the automaton) has size bounded by $|\operatorname{sub}(\mathbf{A}, \mathcal{O})|+1$ since it consists of $|\operatorname{sub}(\mathbf{A}, \mathcal{O})|+1$ lattice values, and

(iii) building a state or a transition requires only to guess $|\operatorname{sub}(\mathbf{A}, \mathcal{O})|+1$ or $k(|\operatorname{sub}(\mathbf{A}, \mathcal{O})|+1)$ lattice values, respectively, and then verifying that this is indeed a valid state or transition of the automaton, which can be done in time polynomial in $|\operatorname{sub}(\mathbf{A}, \mathcal{O})|$.

However, one can easily find runs of $\mathcal{A}_{\mathrm{A}, \mathcal{O}}$ where blocking occurs only after exponentially many transitions, violating the first condition of PSPACE on-the-fly constructions. We will use a faithful family of functions to obtain a reduced 
automaton that guarantees blocking after at most polynomially many transitions, thus obtaining the PSPACE upper bound.

\subsection{Acyclic TBoxes in $L-\mathcal{A L C H \mathcal { I }}$}

In the case of $L-\mathcal{A L C H}$, the faithful family of functions only needs to guarantee that the maximal role depth decreases with each transition. For the acyclic TBox $\mathcal{T}$, the role depth w.r.t. $\mathcal{T}\left(\mathrm{rd}_{\mathcal{T}}\right)$ of concepts is recursively defined as follows:

$-\operatorname{rd}_{\mathcal{T}}(A)=\operatorname{rd}_{\mathcal{T}}(T)=\operatorname{rd}_{\mathcal{T}}(\perp)=0$ for each primitive concept name $A$,

$-\operatorname{rd}_{\mathcal{T}}(A)=\operatorname{rd}_{\mathcal{T}}(C)$ for every definition $\langle A \doteq C, \ell\rangle \in \mathcal{T},{ }^{9}$

$-\operatorname{rd}_{\mathcal{T}}(C \sqcap D)=\operatorname{rd}_{\mathcal{T}}(C \sqcup D)=\max \left\{\operatorname{rd}_{\mathcal{T}}(C), \operatorname{rd}_{\mathcal{T}}(D)\right\}$,

$-\operatorname{rd}_{\mathcal{T}}(C \rightarrow D)=\max \left\{\operatorname{rd}_{\mathcal{T}}(C), \operatorname{rd}_{\mathcal{T}}(D)\right\}$,

$-\operatorname{rd}_{\mathcal{T}}(\neg C)=\operatorname{rd}_{\mathcal{T}}(C)$, and

$-\operatorname{rd}_{\mathcal{T}}(\exists r . C)=\operatorname{rd}_{\mathcal{T}}(\forall r . C)=\operatorname{rd}_{\mathcal{T}}(C)+1$.

We use $\operatorname{rd}_{\mathcal{T}}(H)$ to denote the maximal role depth $\operatorname{rd}_{\mathcal{T}}(D)$ of a concept $D$ in support $(H)$. For a non-negative integer $n$, $\operatorname{sub}^{\leq n}(\mathbf{A}, \mathcal{O})$ denotes the set of all concepts in $\operatorname{sub}(\mathbf{A}, \mathcal{O})$ with role depth less than or equal to $n$.

Definition 5.1 (functions $\left.f_{(H, i)}\right)$ Let $(H, i)$ and $\left(H^{\prime}, i^{\prime}\right)$ be two states of $\mathcal{A}_{\mathbf{A}, \mathcal{O}}$ and $n=\operatorname{rd}_{\mathcal{T}}(H)$. We define the function $f_{(H, i)}\left(H^{\prime}, i^{\prime}\right)=\left(H^{\prime \prime}, i^{\prime}\right)$, where

$H^{\prime \prime}(D)=\left\{\begin{array}{l}H^{\prime}(D) \quad \text { if } D \in \operatorname{sub}^{\leq n-1}(\mathbf{A}, \mathcal{O}), \\ \text { undefined otherwise, }\end{array}\right.$

$H^{\prime \prime}(\rho)= \begin{cases}\mathbf{0} & \text { if } \operatorname{support}(H)=\emptyset, \\ H^{\prime}(\rho) & \text { otherwise. }\end{cases}$

Since $\mathcal{T}$ is acyclic, $H^{\prime \prime}$ as defined above is still a Hintikka function for $\mathbf{A}, \mathcal{O}$ and compatible with all the axioms in $\mathcal{O}$. The proof of the following lemma can be found in the Appendix.

Lemma 5.2 The family $f_{(H, i)}$ from Definition 5.1 is faithful w.r.t. $\mathcal{A}_{\mathbf{A}, \mathcal{O}}$.

By Lemma 2.4, it now follows that $\mathcal{A}_{\mathbf{A}, \mathcal{O}}$ is empty iff the subautomaton $\mathcal{A}_{\mathbf{A}, \mathcal{O}}$ induced by the family $f_{(H, s)}$ is empty. It remains to show that this can be decided in PSPACE.

Lemma 5.3 The construction of $\mathcal{A}_{\mathbf{A}, \mathcal{O}}^{S}$ from a local ABox $\mathbf{A}$ and an ontology $\mathcal{O}=(\mathcal{T}, \mathcal{R})$ with an acyclic TBox $\mathcal{T}$ is a PSPACE on-the-fly construction.

Proof We show that the automata $\mathcal{A}_{\mathbf{A}, \mathcal{O}}^{S}$ are $m$-blocking for $m=\max \left\{\operatorname{rd}_{\mathcal{T}}(D) \mid D \in \operatorname{sub}(\mathbf{A}, \mathcal{O})\right\}+2$, with equality as the blocking relation. The other conditions of Definition 2.5 have already been shown above.

$\overline{9}$ The acyclicity of $\mathcal{T}$ ensures that this is well-defined.
By definition of $\mathcal{A}_{\mathbf{A}, \mathcal{O}}^{S}$, every transition decreases the maximal role depth of the support of the state. Hence, after at $\operatorname{most} \max \left\{\operatorname{rd}_{\mathcal{T}}(D) \mid D \in \operatorname{sub}(\mathbf{A}, \mathcal{O})\right\}$ transitions, we must reach a state $H$ for which $H(D)$ is undefined for all concepts $D \in \operatorname{sub}(\mathbf{A}, \mathcal{O})$, and hence support $(H)=\emptyset$. From the next transition on, all states additionally satisfy that $H(\rho)=\mathbf{0}$. Hence, after at most $m$ transitions, we find two states that are equal. Since $m$ is bounded by a polynomial in the combined size of $\mathbf{A}$ and $\mathcal{O}$, the automata $\mathcal{A}_{\mathbf{A}, \mathcal{O}}^{S}$ satisfy the requirements for a PSPACE on-the-fly construction.

Theorem 2.6 yields the desired PSPACE upper bound for local consistency w.r.t. acyclic TBoxes in the lattice-based description logic $L-\mathcal{A L C H} \mathcal{H}$.

\subsection{Acyclic TBoxes in $L-\mathcal{S} \mathcal{I}_{\mathrm{C}}$}

In the logic $L-\mathcal{S} \mathcal{I}$, we cannot directly reduce the role depth as in the previous section, due to the transitivity conditions. However, if we restrict to crisp roles only, we can still provide a PSPACE upper bound using a faithful family of functions.

Since the interpretations of roles are restricted to have values from $\{\mathbf{0}, \mathbf{1}\}$, all Hintikka functions $H$ now need to satisfy the additional condition that $H(\rho) \in\{\mathbf{0}, \mathbf{1}\}$. It is easy to see that Theorem 4.5 also holds in the presence of this modification. Given a Hintikka function $H$ and a complex role $s$, we define the sets

$$
\begin{aligned}
\left.H\right|_{s} & :=\{D \in \operatorname{support}(H) \mid D \text { is } \exists s . E \text { or } \forall s . E\}, \\
H^{-s} & :=\{D \in \operatorname{support}(H) \mid \exists s . D \text { or } \forall s . D \in \operatorname{sub}(\mathbf{A}, \mathcal{O})\} .
\end{aligned}
$$

Definition 5.4 (functions $\left.g_{(H, i)}\right)$ Let $(H, i)$ and $\left(H^{\prime}, i^{\prime}\right)$ be two states of $\mathcal{A}_{\mathbf{A}, \mathcal{O}}$ and $n=\operatorname{rd}_{\mathcal{T}}(H)$. We define the function $g_{(H, i)}\left(H^{\prime}, i^{\prime}\right)=\left(H^{\prime \prime}, i^{\prime}\right)$, where $i^{\prime}=\varphi(E)$ for $E \in \operatorname{sub}(\mathbf{A}, \mathcal{O})$ of the form $\exists$ s.F or $\forall s . F$,

$$
\begin{aligned}
P & = \begin{cases}\left.\operatorname{sub}^{\leq n}(\mathbf{A}, \mathcal{O}) \cap H^{\prime}\right|_{s} & \text { if } s \text { is transitive, } \\
\text { otherwise, }\end{cases} \\
H^{\prime \prime}(D) & = \begin{cases}H^{\prime}(D) & \text { if } D \in \text { sub }^{\leq n-1}(\mathbf{A}, \mathcal{O}) \cup P, \\
\text { undefined } & \text { otherwise, }\end{cases} \\
H^{\prime \prime}(\rho) & = \begin{cases}\mathbf{0} & \text { if support }(H)=\emptyset, \\
H^{\prime}(\rho) \text { otherwise. }\end{cases}
\end{aligned}
$$

These functions are a natural generalization of the functions used in [2] to provide a PSPACE upper bound for crisp $\mathcal{S I}$. The proof of the following lemma can also be found in the Appendix.

\section{Lemma 5.5 The family $g_{(H, s)}$ from Definition 5.4 is faithful} w.r.t. $\mathcal{A}_{\mathbf{A}, \mathcal{O}}$.

To show that the automata $\mathcal{A}_{\mathbf{A}, \mathcal{O}}^{S}$ can be built by a PSPACE on-the-fly construction, we employ the following blocking relation $\longleftarrow \mathcal{S I}_{\mathrm{c}}$. 
Definition $5.6\left(\leftarrow \mathcal{S I}_{c}\right)$ Let $(H, i)$ and $\left(H^{\prime}, i^{\prime}\right)$ be two states of $\mathcal{A}_{\mathbf{A}, \mathcal{O}}^{S}$. We define $(H, i) \longleftarrow \mathcal{S I}_{\mathrm{c}}\left(H^{\prime}, i^{\prime}\right)$ iff $i=i^{\prime}=\varphi(E)$ for $E \in \operatorname{sub}(\mathbf{A}, \mathcal{O})$ of the form $\exists$ s.F or $\forall s . F$ and either

(i) $H=H^{\prime}$,

(ii) $H(\rho)=H^{\prime}(\rho)=\mathbf{0}$ and the sets $\left.\left.H\right|_{s} \cup H^{-s} \cup H\right|_{\bar{s}}$ and $\left.\left.H^{\prime}\right|_{s} \cup H^{\prime-s} \cup H^{\prime}\right|_{\bar{s}}$ are equal, or

(iii) 1. $s$ is transitive, $H(\rho)=H^{\prime}(\rho)=\mathbf{1}, H(F)=H^{\prime}(F)$, 2. $H(D)=H^{\prime}(D)$ for every concept $D$ in

$$
\mathcal{Q}\left(H, H^{\prime}, s\right):=\left.\left.\left.\left.H\right|_{s} \cup H^{\prime}\right|_{s} \cup H\right|_{\bar{s}} \cup H^{\prime}\right|_{\bar{s}} \text {, and }
$$

3. we have $H^{\prime}(G) \leq H^{\prime}(\exists s . G)$ for every $\exists s .\left.G \in H^{\prime}\right|_{s}$ and $H^{\prime}(G) \geq H^{\prime}(\forall s . G)$ for every $\forall s .\left.G \in H^{\prime}\right|_{s}$.

To see that the automata $\mathcal{A}_{\mathbf{A}, \mathcal{O}}^{S}$ are $\longleftarrow \mathcal{S} \mathcal{I}_{\mathrm{c}}$-invariant, we analyze the three conditions above:

(i) The equality relation trivially satisfies the notion of $\longleftarrow \mathcal{S \mathcal { I } _ { \mathrm { c } }}$-invariance.

(ii) Recall that if $H(\rho)=\mathbf{0}$, then all the inequalities in the Conditions b), c), b') and c') from Definition 4.4 are satisfied. Furthermore, conditions a) and a') remain satisfied when replacing one successor $H$ of $H_{0}$ with $H(\rho)=\mathbf{0}$ by $H^{\prime}$ which also satisfies $H^{\prime}(\rho)=\mathbf{0}$. Thus, one only needs to ensure that $H^{\prime}$ is defined for the relevant concepts, which is expressed by the second part of this condition.

(iii) The first condition ensures that Conditions a) and a') of Definition 4.4 remain satisfied. The second condition restricts all the quantified concepts that are transferred by the transitive role $s$ to be evaluated by identical values. Thus, Conditions c) and c') and the last inequalities of Condition b) and b') of Definition 4.4 are still satisfied. Finally, the third condition ensures that the first inequalities from Conditions b) and b') are satisfied: Since $H_{0}(\forall s . G) \leq H^{\prime}(\rho) \Rightarrow H^{\prime}(\forall s . G)$ is already satisfied, the additional condition $H^{\prime}(\forall s . G) \leq H^{\prime}(G)$ ensures that also $H_{0}(\forall s . G) \leq H^{\prime}(\rho) \Rightarrow H^{\prime}(G)$ holds, and dually for the existential restrictions.

That the subautomata are polynomially blocking w.r.t. $\longleftarrow \mathcal{S I}_{\mathrm{C}}$ can be shown using the following facts:

- The role depth of the Hintikka sets strictly decreases along non-transitive role connections. The same is true if on two consecutive transitions two different roles are used, regardless of whether they are transitive or not.

- For a chain of successors $H_{0}, H_{1}, \ldots$ through the same transitive role $s$, we have $\left.\left.H_{i}\right|_{s} \subseteq H_{i+1}\right|_{s}$ and $\left.\left.H_{i}\right|_{\bar{s}} \supseteq H_{i+1}\right|_{\bar{s}}$ for all $i \geq 0$. Additionally, if $H_{i+1}(\rho)=\mathbf{1}$, then we have $H_{i}(\forall s . G) \leq H_{i+1}(\forall s . G)$ and $H_{i}(\exists s . G) \geq H_{i+1}(\exists s . G)$ for any $\forall s . G \in \operatorname{sub}(\mathbf{A}, \mathcal{O})$ or $\exists s . G \in \operatorname{sub}(\mathbf{A}, \mathcal{O})$, respectively.

It is important to point out that this last argument only holds when the degree of the role relation between successors is exactly $\mathbf{1}$. Thus, this proof cannot be used to prove a polynomial bound on blocking for the more general fuzzy DL $L-\mathcal{S I}$. The full details can be found in the appendix.

Lemma 5.7 The construction of $\mathcal{A}_{\mathbf{A}, \mathcal{O}}^{S}$ from a local ABox $\mathbf{A}$ and an ontology $\mathcal{O}=(\mathcal{T}, \mathcal{R})$ with an acyclic TBox $\mathcal{T}$ is a PSPACE on-the-fly construction.

As before, this yields a PSPACE upper bound for local consistency in $L-\mathcal{S} \mathcal{I}_{c}$. Thus, local consistency, strong satisfiability, and subsumption w.r.t. acyclic TBoxes are PSPACEcomplete in $L-\mathcal{A} \mathcal{L C H} \mathcal{I}$ and $L-\mathcal{S} \mathcal{I}_{\mathrm{c}}$. This follows from the fact that these problems are already PSPACE-hard in $\mathcal{A L C}$ w.r.t. the empty TBox [29].

Theorem 5.8 In all logics above $\mathcal{A L C}$ and below $L-S \mathcal{I}_{\mathrm{C}}$ or $L-\mathcal{A L C H}$, deciding local consistency, strong satisfiability, and subsumption w.r.t. acyclic TBoxes is PSPACE-complete.

In particular, if we restrict to crisp $\mathcal{A L C H} \mathcal{L}$, we obtain a previously unknown, although not surprising result.

Corollary 5.9 In $\mathcal{A} \mathcal{L C H}$, satisfiability and subsumption w.r.t. acyclic TBoxes can be decided in PSPACE.

\section{Dealing with Different Semantics and Extensions}

The constructions used in the previous sections provide a mechanism for reasoning w.r.t. witnessed models. This is in fact a restriction, since most fuzzy DLs do not have the witnessed model property (recall Example 4.2). However, Lemma 4.1 ensures the existence of a finite $n$, which depends exclusively on the lattice $L$, such that $L-\mathcal{S H} \mathcal{I}$ has the $n$-witnessed model property.

One can build analogous automata for reasoning w.r.t. $n$-witnessed models, for any $n \in \mathbb{N}$. To do this, one needs to consider $(n k)$-ary automata, where $n$ successors are used to witness each of the quantified concepts in $\operatorname{sub}(\mathbf{A}, \mathcal{O})$. Thus, the arity of these automata grows polynomially in $n$. Moreover, the blocking relations and limits on the lengths paths may have before blocking is triggered remain unchanged. This means that the complexity results from Theorems 4.7 and 5.8 still hold.

Up to now, we have considered the lattice $L$ to be fixed. We now want to shortly analyze the influence of $L$ on the complexity results. We first assume that $L$ is given as an explicit list of all its elements and that the lattice operations $\vee, \wedge, \sim, \otimes, \oplus, \Rightarrow$ can be computed in polynomial time in the 
size of the given lattice elements. Under these assumptions, the construction of the Hintikka automata in Sect. 4 is still exponential since these automata have $(|L|+1)^{|\operatorname{sub}(\mathbf{A}, \mathcal{O})|+1}$ states, where $|L|$ is the cardinality of $L$, which is polynomial in the size of the input.

For the results of Sect. 5, we observe the following:

(i) the arity of the automata is not affected by $L$,

(ii) every Hintikka function is $(|\operatorname{sub}(\mathbf{A}, \mathcal{O})|+1)$-times the size of a single lattice element, and thus still polynomial in the size of the input, and

(iii) guessing one or $k+1$ Hintikka functions can thus be done in polynomial time and verifying the conditions of Definitions 4.3 and 4.4 is also possible in polynomial time by our assumption on the complexity of the lattice operations.

To conclude, it can be seen from the proofs of Lemmata 5.3 and 5.7 that the maximal length of non-blocked paths in the respective subautomata is also bounded polynomially in the size of the input. In fact, the bound used in the proof of Lemma 5.3 does not depend on $L$ at all. Thus, also the complexity results of Sect. 5 are not affected by $L$.

If the cardinality $|L|$ is exponential in the size of the input representation of $L$, then all complexity results except Lemma 5.7 still hold. The bound established in the proof of this lemma would then be exponential. If $|L|$ is even doubly exponential in the input, then also the other results do not hold anymore. However, as mentioned before, we think that for application purposes one can view the lattice $L$ as fixed since it will be specified in the beginning and then used in all ontologies.

We also point out that our semantics is general enough to cover other seemingly different approaches. First, one could think of extending the notion of acyclic TBoxes to allow also axioms of the form $\langle A \sqsubseteq C, \ell\rangle$, as long as the acyclicity condition is still satisfied.

Lemma 6.1 The $G C I\langle A \sqsubseteq C, \ell\rangle$ is equivalent to the concept definition $\langle A \doteq C \sqcap D, \ell\rangle$, where $D$ is a new concept name, if the $t$-norm is continuous.

Regarding the interpretation of the constructors, different ideas have been considered in the literature. On the one hand, in Mathematical Fuzzy Logic, negation is usually interpreted through the residual negation defined as $\ell \Rightarrow \mathbf{0}$ for all $\ell \in L$. This operator can be expressed by the concept construction $C \rightarrow \perp$. On the other hand, GCIs are sometimes interpreted using a different kind of implication, which is defined as $\sim \ell_{1} \oplus \ell_{2}$. For the Gödel $t$-norm, this is called the KleeneDienes implication. This semantics can be simulated in our approach by the axiom $\langle\top \sqsubseteq \neg C \sqcup D, \ell\rangle$.
This shows that our complexity upper bounds also apply to the syntactic and semantic variants of fuzzy DLs over lattices that have been studied previously.

We have so far focused only on deciding local consistency. In general, one is often interested in deciding consistency of general ABoxes, which can express restrictions between several individuals, and their role connections $[9,30]$. As it was shown in [15], if local consistency is NP-hard, then consistency of general ABoxes belongs to the same complexity class as local consistency. In particular, this implies that for the logics studied in this paper, deciding general consistency is as hard as local consistency.

Corollary 6.2 Consistency of general ABoxes in L-SH and $L-\mathcal{S H I}$ with or without TBoxes is EXPTIME-complete.

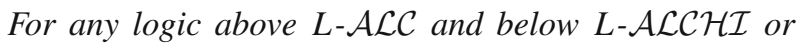
$L-\mathcal{S} \mathcal{I}_{\mathrm{c}}, A B o x$ consistency is PSPACE-complete if the TBox is acyclic, and EXPTIME-complete otherwise.

\section{Conclusions}

We have studied the complexity of reasoning in $L-\mathcal{S H} \mathcal{I}$ and several of its sublogics and shown that the semantics based on finite residuated De Morgan lattices does not make reasoning harder compared to their crisp versions. More precisely, strong satisfiability and subsumption in $L-\mathcal{S H} \mathcal{I}$ w.r.t. general TBoxes can be decided in exponential time. This yields ExPTIME-completeness for reasoning in this logic, even if the TBox is empty. For this, we generalized the results from [2] to deal with fuzzy concepts and roles and a role hierarchy.

If we restrict to acyclic TBoxes in the logics $L-\mathcal{A L C H} \mathcal{I}$ and $L-S \mathcal{I}_{\mathrm{C}}$, then we obtain a PSPACE upper bound. This extends the complexity results that have previously been obtained for special cases of these logics $[12,13,17]$. The construction of the faithful family of functions for $L-\mathcal{S} \mathcal{I}_{\mathrm{C}}$ is obtained by generalizing the one from [2] to allow a fuzzy interpretation of concepts.

Furthermore, combining the obtained results with the tableau construction from [15] shows that the same complexity bounds also hold for the more general consistency problem with arbitrary ABoxes. The results are summarized in Table 1. As can be easily seen from the table, the complexity of reasoning with semantics based on finite lattices matches the known complexity bounds for crisp reasoning. Recall that under crisp semantics, $\mathcal{S}_{\mathrm{C}} / \mathcal{S} \mathcal{I}_{\mathrm{C}}$ and $\mathcal{S} / \mathcal{S I}$ denote the same logics.

To implement the presented algorithms, one would first need to find a smart way to deal with the blow-up that comes from the enumeration of all combinations of lattice values. In this respect, our approach suffers from the same problems as previous reductions to crisp reasoning [10,11]. However, 
Table 1 The complexity of reasoning in the DLs between $\mathcal{A L C}$ and $\mathcal{S H \mathcal { I }}$ with crisp and finitely-valued fuzzy semantics.

\begin{tabular}{llllll}
\hline base logic & \multicolumn{2}{l}{ acyclic TBox } & & \multicolumn{2}{l}{ general TBox } \\
\cline { 2 - 3 } \cline { 5 - 6 } & crisp & finite $L$ & & crisp & finite $L$ \\
\hline$-\mathcal{A L C H} \mathcal{H}$ & PSPACE & PSPACE & & EXPTIME & EXPTIME \\
$\mathcal{S}_{\mathrm{C}}-\mathcal{S} \mathcal{I}_{\mathrm{C}}$ & PSPACE & PSPACE & & EXPTIME & EXPTIME \\
$\mathcal{S}-\mathcal{S} \mathcal{I}$ & PSPACE & $?$ & EXPTIME & EXPTIME \\
$\mathcal{S H}-$ & EXPTIME & EXPTIME & & EXPTIME & EXPTIME \\
\hline
\end{tabular}

for the special case of Zadeh semantics, these reductions are polynomial $[10,30]$, and much better suited for implementation than an automata-based approach. The main advantage of our technique is that it allows us to obtain tight complexity bounds for the case of an arbitrary finite lattice.

Our analysis leaves open the precise complexity of reasoning in $L-\mathcal{S} \mathcal{I}$ w.r.t. acyclic TBoxes and fuzzy roles. While it is possible that similar techniques as in Sect. 5.2 can be used to show a PSPACE upper bound, the argument used in this paper fails whenever roles are not crisp. In fact, even if roles were allowed to assume just one value $\ell$ different from $\mathbf{0}$ and 1, we would obtain only an exponential bound for blocking. We also did not consider labeled role inclusions $\langle r \sqsubseteq s, \ell\rangle$ that allow to specify an inclusion degree as in GCIs. We plan to cover these issues in future work.

We also plan to extend our complexity analysis to more complex fuzzy DLs, such as $L-\mathcal{S} \mathcal{R O} \mathcal{I} \mathcal{Q}$. This is not a trivial task for several reasons. First of all, it is not clear how qualified cardinality restrictions $(\mathcal{Q})$ should be defined in the absence of the witnessed model property: if an existential restriction $\exists r . C$ can only be satisfied using three successors, then what is the meaning of an expression of the form $(\leq 2$ r.C) ? Following the semantics proposed in [11] for finite-valued Lukasiewicz semantics w.r.t. witnessed models, this concept could never have a value of $\mathbf{1}$. However, it might be desirable to evaluate it "modulo" the number of witnesses needed to produce the value of $\exists r$.C. In addition, complex role inclusion axioms $(\mathcal{R})$ and nominals $(\mathcal{O})$ make the use of tree-like structures, and hence of tree automata, much harder. For these cases, we plan to develop a tableauxbased decision procedure that can produce general graph-like models.

\section{Appendix A: Proofs}

This appendix contains some of the more technical proofs for the results presented in the paper.

Proof of Theorem 4.5 To improve readability, we prove Theorem 4.5 using three lemmata. Lemma A.1 provides an auxiliary result that allows us to restrict the remaining arguments to acyclic RBoxes. Lemmata A.2 and A.3 then show the two directions of Theorem 4.5.

Lemma A.1 Local consistency in $L-\mathcal{S H I}$ is polynomially reducible to local consistency in $L-\mathcal{S H I}$ w.r.t. acyclic RBoxes. This also holds when the TBox is restricted to be acyclic.

Proof This claim can be shown similarly to the corresponding result for crisp $\mathcal{S H \mathcal { I }}$ [31]. Let $\mathbf{A}=$ $\left\{\left(C_{1}, \ell_{1}\right), \ldots,\left(C_{m}, \ell_{m}\right)\right\}$ be a local ABox and $\mathcal{O}=(\mathcal{T}, \mathcal{R})$ an ontology. One can construct an acyclic RBox $\mathcal{R}^{\prime}$ from $\mathcal{R}$ in polynomial time as follows. Consider the role hierarchy $\sqsubseteq \mathcal{R}$ as a directed graph. One can find the strongly connected components of this graph in quadratic time. For each such component $\left\{s_{1}, \ldots, s_{n}\right\}$, choose a representative $s$ such that $s$ is transitive whenever at least one role of $\left\{s_{1}, \ldots, s_{n}\right\}$ is transitive.

It is easy to see that for every component $\left\{s_{1}, \ldots, s_{n}\right\}$ and every model $\mathcal{I}$ of $\mathcal{O}$, we have $s_{i}^{\mathcal{I}}=s_{j}^{\mathcal{I}}$ for all $i, j \in\{1, \ldots, n\}$. Furthermore, if this component contains a transitive role, then all $s_{i}^{\mathcal{I}}$ are transitive. The local ABox $\mathbf{A}^{\prime}$ and the ontology $\mathcal{O}^{\prime}=\left(\mathcal{T}^{\prime}, \mathcal{R}^{\prime}\right)$ are created from $\mathbf{A}$ and $\mathcal{O}$ by replacing each role $s$ by the representative chosen for its strongly connected component. Thus, whenever $\mathbf{A}$ is locally consistent w.r.t. $\mathcal{O}$, then $\mathbf{A}^{\prime}$ is locally consistent w.r.t. $\mathcal{O}^{\prime}$, and vice versa. Finally, note that $\mathcal{T}^{\prime}$ is acyclic iff $\mathcal{T}$ is.

In the following, let $\mathbf{A}$ be a local $\mathrm{ABox}$ and $\mathcal{O}=(\mathcal{T}, \mathcal{R})$ an ontology with an acyclic RBox $\mathcal{R}$.

Lemma A.2 If there is a Hintikka tree $\mathbf{T}$ for $\mathbf{A}, \mathcal{O}$ with $\mathbf{T}(\varepsilon)(C)=\ell$ for all $(C, \ell) \in \mathbf{A}$, then $\mathbf{A}$ is locally consistent w.r.t. $\mathcal{O}$.

Proof We will define a model $\mathcal{I}$ of $\mathcal{O}$ over the domain $\Delta^{\mathcal{I}}=K^{*}$ such that $C^{\mathcal{I}}(\varepsilon)=\ell$ for every $(C, \ell) \in \mathbf{A}$. For a role name $r$, we define the fuzzy binary relation $r^{\mathbf{T}}$ on $\Delta^{\mathcal{I}}$ by

$-r^{\mathbf{T}}(x, x \varphi(E))=\mathbf{T}(x \varphi(E))(\rho)$ if $E \in \operatorname{sub}(\mathbf{A}, \mathcal{O})$ is of the form $\exists s . F$ or $\forall s . F$ for some $s \sqsubseteq \mathcal{R} r$;

- $r^{\mathbf{T}}(x \varphi(E), x)=\mathbf{T}(x \varphi(E))(\rho)$ if $E \in \operatorname{sub}(\mathbf{A}, \mathcal{O})$ of the form $\exists$ s.F or $\forall s . F$ for some $s \sqsubseteq \mathcal{R} \bar{r}$;

$-r^{\mathbf{T}}(x, y)=\mathbf{0}$, otherwise. 
For $x_{1}, \ldots, x_{n} \in \Delta^{\mathcal{I}}$, we define

$r^{\mathbf{T}}\left(x_{1}, \ldots, x_{n}\right)=r^{\mathbf{T}}\left(x_{1}, x_{2}\right) \otimes \ldots \otimes r^{\mathbf{T}}\left(x_{n-1}, x_{n}\right)$.

We define the interpretation of a role name $r$ as follows. ${ }^{10}$ If $r$ is transitive, $r^{\mathcal{I}}$ is the transitive closure of $r^{\mathbf{T}}$, i.e. for all $x, y \in \Delta^{\mathcal{I}}$,

$r^{\mathcal{I}}(x, y)=\bigvee_{n \geq 0} \bigvee_{z_{1}, \ldots, z_{n} \in \Delta^{\mathcal{I}}} r^{\mathbf{T}}\left(x, z_{1}, \ldots, z_{n}, y\right)$.

If $r$ is not transitive, we define

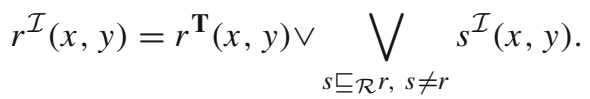

This complex expression is necessary to correctly account for the transitive sub-roles of $r$. It is well-defined since $\mathcal{R}$ is acyclic. This definition can be summarized as

$$
\begin{aligned}
& r^{\mathcal{I}}(x, y)=r^{\mathbf{T}}(x, y) \vee \\
& \bigvee_{\substack{s \subseteq \mathcal{R} r \\
s \text { transitive }}} \bigvee_{n \geq 1} \bigvee_{z_{1}, \ldots, z_{n} \in \Delta^{\mathcal{I}}} s^{\mathbf{T}}\left(x, z_{1}, \ldots, z_{n}, y\right) .
\end{aligned}
$$

It is easy to show that the same equations hold for an inverse role $s=r^{-}$if we define $s^{\mathbf{T}}(x, y)=r^{\mathbf{T}}(y, x)$ for all $x, y \in \Delta^{\mathcal{I}}$ and

$s^{\mathbf{T}}\left(x_{1}, \ldots, x_{n}\right)=s^{\mathbf{T}}\left(x_{1}, x_{2}\right) \otimes \ldots \otimes s^{\mathbf{T}}\left(x_{n-1}, x_{n}\right)$

for all $x_{1}, \ldots, x_{n} \in \Delta^{\mathcal{I}}$.

For a primitive concept name $A$, we set $A^{\mathcal{I}}(x)=\mathbf{T}(x)(A)$ for all $x \in \Delta^{\mathcal{I}}$. To show that $\mathcal{I}$ can be extended to defined concept names such that it agrees with $\mathbf{T}$ on complex concepts, we define a weight function $o(D)$ for concepts:

- $o(T)=o(\perp)=o(A)=0$ for a primitive concept name $A$;

$-o(B)=o(D)+1$ if $\langle B \doteq D, m\rangle \in \mathcal{T}$;

$-o(\neg D)=o(D)+1$;

$-o(D \sqcap E)=o(D \sqcup E)=o(D \rightarrow E)=\max \{o(D)$, $o(E)\}+1$;

- $o(\exists r . D)=o(\forall r . D)=o(D)+1$.

If there are concept definitions in $\mathcal{T}$, then they have an acyclic dependency relation. Thus, the order on concepts induced by their weights is well-founded. We show by induction on the weight of $D$ that, if $\mathbf{T}(x)(D)$ is defined for some $x \in \Delta^{\mathcal{I}}$, then $D^{\mathcal{I}}(x)=\mathbf{T}(x)(D)$ :

- Primitive concept names, $\top$, and $\perp$ are interpreted correctly by the definition of $\mathcal{I}$ and Hintikka sets.

\footnotetext{
$\overline{10}$ This definition is based on the construction for crisp $\mathcal{S H \mathcal { I }}$ [24].
}

- If $\mathbf{T}(x)(B)$ is defined for a concept name $B$ with $\langle B \doteq D, m\rangle \in \mathcal{T}$, since $\mathbf{T}(x)$ is compatible with $\langle B \doteq D, m\rangle, \mathbf{T}(x)(D)$ is also defined. Furthermore, $(\mathbf{T}(x)(B) \Rightarrow \mathbf{T}(x)(D)) \otimes(\mathbf{T}(x)(D) \Rightarrow \mathbf{T}(x)(B)) \geq m$. Since $o(D)<o(B)$, we have $D^{\mathcal{I}}(x)=\mathbf{T}(x)(D)$ by induction. Thus, defining $B^{\mathcal{I}}(x)=\mathbf{T}(x)(B)$ ensures that $\mathcal{I}$ satisfies $\langle B \doteq D, m\rangle$.

- If $\mathbf{T}(x)(\neg D)$ is defined, then $\mathbf{T}(x)(D)$ is also defined. By induction, we have $(\neg D)^{\mathcal{I}}(x)=\sim D^{\mathcal{I}}(x)=$ $\sim \mathbf{T}(x)(D)=\mathbf{T}(x)(\neg D)$.

- The claims for $D \sqcap E, D \sqcup E$ and $D \rightarrow E$ follow similarly.

- If $\mathbf{T}(x)(\exists r . D)=m$, let $y:=x \varphi(\exists r . D)$. By the Hintikka condition, $\mathbf{T}(y)(D)$ is defined, and by induction $D^{\mathcal{I}}(y)=\mathbf{T}(y)(D)$. Moreover,

$$
\begin{aligned}
m & =\mathbf{T}(y)(\rho) \otimes \mathbf{T}(y)(D)=r^{\mathbf{T}}(x, y) \otimes D^{\mathcal{I}}(y) \\
& \leq r^{\mathcal{I}}(x, y) \otimes D^{\mathcal{I}}(y) .
\end{aligned}
$$

We now show that for every $z \in \Delta^{\mathcal{I}}, r^{\mathcal{I}}(x, z) \otimes D^{\mathcal{I}}(z)$ $\leq m$ holds. This in particular implies that $y$ is a witness for $(\exists r . D)^{\mathcal{I}}(x)$. By definition of $r^{\mathcal{I}}$ and monotonicity of $\otimes$, it suffices to show that
(a) $r^{\mathbf{T}}(x, z) \otimes D^{\mathcal{I}}(z) \leq m$ and
(b) $s^{\mathbf{T}}\left(x, y_{1}, \ldots, y_{n}, z\right) \otimes D^{\mathcal{I}}(z) \leq m$

for all transitive roles $s \sqsubseteq \mathcal{R}^{r}$ and all $z_{1}, \ldots, z_{n} \in \Delta^{\mathcal{I}}$, $n \geq 1$.

a) If $r^{\mathbf{T}}(x, z)=\mathbf{0}$, the claim is trivial; otherwise, there must be a restriction $E \in \operatorname{sub}(\mathbf{A}, \mathcal{O})$ of the form $\exists s^{\prime} . F$ or $\forall s^{\prime} . F$ such that either $z=x \varphi(E)$ and $s^{\prime} \sqsubseteq \mathcal{R} r$ or $x=z \varphi(E)$ and $s^{\prime} \sqsubseteq \mathcal{R} \bar{r}$. In the first case, by the Hintikka condition $\mathbf{T}(z)(D)$ is defined and $m=\mathbf{T}(x)(\exists r . D) \geq \mathbf{T}(z)(\rho) \otimes \mathbf{T}(z)(D)=$ $r^{\mathbf{T}}(x, z) \otimes D^{\mathcal{I}}(z)$, by induction. In the second case, the Hintikka condition implies that $\mathbf{T}(z)(D)$ is defined and

$$
\begin{aligned}
m & =\mathbf{T}(x)(\exists r . D) \geq \mathbf{T}(x)(\rho) \otimes \mathbf{T}(z)(D) \\
& =r^{\mathbf{T}}(x, z) \otimes D^{\mathcal{I}}(z) .
\end{aligned}
$$

b) Again, the claim for $s^{\mathbf{T}}\left(x, y_{1}, \ldots, y_{n}, z\right)=\mathbf{0}$ is trivial. If this is not the case, then $s^{\mathbf{T}}\left(x, y_{1}\right), s^{\mathbf{T}}\left(y_{1}, y_{2}\right)$, $\ldots, s^{\mathbf{T}}\left(y_{n-1}, y_{n}\right), s^{\mathbf{T}}\left(y_{n}, z\right)$ are all greater than 0. Since $s^{\mathbf{T}}\left(x, y_{1}\right)>\mathbf{0}$, there must be a restriction $E \in \operatorname{sub}(\mathbf{A}, \mathcal{O})$ of the form $\exists s^{\prime} . F$ or $\forall s^{\prime} . F$ such that either $y_{1}=x \varphi(E)$ and $s^{\prime} \sqsubseteq \mathcal{R} s$ or $x=$ $y_{1} \varphi(E)$ and $s^{\prime} \sqsubseteq \mathcal{R} \bar{s}$. In the first case, we have $\mathbf{T}(x)(\exists r . D) \geq \mathbf{T}\left(y_{1}\right)(\rho) \otimes \mathbf{T}\left(y_{1}\right)(\exists s . D)$; in the second case, $\mathbf{T}(x)(\exists r . D) \geq \mathbf{T}(x)(\rho) \otimes \mathbf{T}\left(y_{1}\right)(\exists s . D)$. 
Thus, in both cases it holds that $\mathbf{T}(x)(\exists r . D) \geq$ $s^{\mathbf{T}}\left(x, y_{1}\right) \otimes \mathbf{T}\left(y_{1}\right)(\exists s . D)$. Analogously, one can show that for every $i \in\{1, \ldots, n-1\}, \mathbf{T}\left(y_{i}\right)(\exists s . D)$ $\geq s^{\mathbf{T}}\left(y_{i}, y_{i+1}\right) \otimes \mathbf{T}\left(y_{i+1}\right)(\exists s . D)$ holds, and additionally as in case a), $\mathbf{T}\left(y_{n}\right)(\exists s . D) \geq s^{\mathbf{T}}\left(y_{n}, z\right)$ $\otimes D^{\mathcal{I}}(z)$. This implies that $m=\mathbf{T}(x)(\exists r . D) \geq$ $s^{\mathbf{T}}\left(x, y_{1}, \ldots, y_{n}, z\right) \otimes D^{\mathcal{I}}(z)$ by monotonicity of $\otimes$.

- The case of $\forall r . D$ can be handled similarly to the previous case since dual Hintikka conditions hold for the value restrictions.

Thus, $\mathcal{I}$ is an interpretation that satisfies all concept definitions in $\mathcal{T}$. We now show that $\mathcal{I}$ satisfies every GCI $\langle D \sqsubseteq E, m\rangle \in \mathcal{T}$. Since every Hintikka set in $\mathbf{T}$ must be compatible with this GCI, $\mathbf{T}(x)(D)$ and $\mathbf{T}(x)(E)$ are always defined and we have $\mathbf{T}(x)(D) \Rightarrow \mathbf{T}(x)(E) \geq m$ for every $x \in K^{*}$. By construction, we thus have $D^{\mathcal{I}}(x) \Rightarrow E^{\mathcal{I}}(x) \geq m$ for every $x \in \Delta^{\mathcal{I}}$, i.e. $\mathcal{I}$ satisfies this GCI.

Consider now a role inclusion $s \sqsubseteq s^{\prime} \in \mathcal{R}$ and $x, y \in \Delta^{\mathcal{I}}$. By definition of $s^{\mathbf{T}}$ and $s^{\prime \mathbf{T}}$, we have $s^{\mathbf{T}}(x, y) \leq s^{\prime \mathbf{T}}(x, y)$. Since every transitive role $r$ with $r \sqsubseteq \mathcal{R} s$ also satisfies $r \sqsubseteq \mathcal{R} s^{\prime}$, we have $s^{\mathcal{I}}(x, y) \leq s^{\prime \mathcal{I}}(x, y)$.

We have thus shown that $\mathcal{I}$ is a model of $\mathcal{O}$. Moreover, for every $(C, \ell) \in \mathbf{A}$ we have $C^{\mathcal{I}}(\varepsilon)=\mathbf{T}(\varepsilon)(C)=\ell$. Thus, $\mathbf{A}$ is locally consistent w.r.t. $\mathcal{O}$.

It remains to show the other direction of Theorem 4.5

Lemma A.3 If $\mathbf{A}$ is locally consistent w.r.t. $\mathcal{O}$, then there is a Hintikka tree $\mathbf{T}$ for $\mathbf{A}, \mathcal{O}$ with $\mathbf{T}(\varepsilon)(C)=\ell$ for all $(C, \ell) \in \mathbf{A}$.

Proof Let $\mathcal{I}$ be a model of $\mathcal{O}$ and $x \in \Delta^{\mathcal{I}}$ such that for every $(C, \ell) \in \mathbf{A}, C^{\mathcal{I}}(x)=\ell$. By our assumption on $L$, $\mathcal{I}$ is witnessed. We show that $\mathcal{I}$ can be "unraveled" into a Hintikka tree $\mathbf{T}$ for $\mathbf{A}, \mathcal{O}$ with $\mathbf{T}(\varepsilon)(C)=\ell$. For this, we inductively define a mapping $g: K^{*} \rightarrow \Delta^{\mathcal{I}}$ that will specify which elements of $\Delta^{\mathcal{I}}$ are represented by the nodes of $\mathbf{T}$. We begin with $g(\varepsilon):=x$.

Let now $v \in K^{*}$ be such that $g(v)$ has already been defined. We set $\mathbf{T}(v)(D):=D^{\mathcal{I}}(g(v))$ for each $D \in \operatorname{Sub}(\mathbf{A}, \mathcal{O})$. Since $\mathcal{I}$ satisfies $\mathcal{T}$, this defines a Hintikka function that is compatible with all axioms of $\mathcal{T}$. For each restriction $\exists r . D \in \operatorname{sub}(\mathbf{A}, \mathcal{O})$, there is a witness $y \in \Delta^{\mathcal{I}}$ with $(\exists r . D)^{\mathcal{I}}(g(v))=r^{\mathcal{I}}(g(v), y) \otimes D^{\mathcal{I}}(y)$. We set $g(\nu \varphi(\exists r . D)):=y$ and $\mathbf{T}(\nu \varphi(\exists r . D))(\rho):=r^{\mathcal{I}}(g(\nu), y)$. For every $\forall r . D \in \operatorname{sub}(\mathbf{A}, \mathcal{O})$, there is a $y \in \Delta^{\mathcal{I}}$ such that $(\forall r . D)^{\mathcal{I}}(g(\nu))=r^{\mathcal{I}}(g(\nu), y) \Rightarrow D^{\mathcal{I}}(y)$ and we define $g(\nu \varphi(\forall r . D)):=y$ and $\mathbf{T}(\nu \varphi(\forall r . D))(\rho):=r^{\mathcal{I}}(g(\nu), y)$.

We show that $\mathbf{T}$ satisfies the Hintikka condition at every transition $(\mathbf{T}(v), \mathbf{T}(v 1), \ldots, \mathbf{T}(v k))$. Let $\exists s . G \in \operatorname{sub}(\mathbf{A}, \mathcal{O})$ and $\mu:=\nu \varphi(\exists s . G)$. Then $\mathbf{T}(\mu)(G)$ is defined by construction and

$$
\begin{aligned}
\mathbf{T}(\nu)(\exists s . G) & =(\exists s . G)^{\mathcal{I}}(g(\nu)) \\
& =r^{\mathcal{I}}(g(\nu), g(\mu)) \otimes G^{\mathcal{I}}(g(\mu)) \\
& =\mathbf{T}(\mu)(\rho) \otimes \mathbf{T}(\mu)(G) .
\end{aligned}
$$

Let now $E \in \operatorname{sub}(\mathbf{A}, \mathcal{O})$ be of the form $\exists s^{\prime} . F$ or $\forall s^{\prime} . F$ with $s^{\prime} \sqsubseteq \mathcal{R} s$ and $\mu^{\prime}:=\nu \varphi(E)$. Then $\mathbf{T}\left(\mu^{\prime}\right)(G)$ is defined by construction and we have

$$
\begin{aligned}
\mathbf{T}(\nu)(\exists s . G) & =(\exists s . G)^{\mathcal{I}}(g(v)) \\
& \geq s^{\mathcal{I}}\left(g(v), g\left(\mu^{\prime}\right)\right) \otimes G^{\mathcal{I}}\left(g\left(\mu^{\prime}\right)\right) \\
& \geq s^{\prime \mathcal{I}}\left(g(\nu), g\left(\mu^{\prime}\right)\right) \otimes G^{\mathcal{I}}\left(g\left(\mu^{\prime}\right)\right) \\
& =\mathbf{T}\left(\mu^{\prime}\right)(\rho) \otimes \mathbf{T}\left(\mu^{\prime}\right)(G) .
\end{aligned}
$$

If $r$ is a transitive role with $s^{\prime} \sqsubseteq \mathcal{R} r \sqsubseteq \mathcal{R} s$, then

$$
\begin{aligned}
\mathbf{T}(v) & (\exists s . G)=(\exists s . G)^{\mathcal{I}}(g(v)) \\
= & \bigvee_{y \in \Delta^{\mathcal{I}}} s^{\mathcal{I}}(g(v), y) \otimes G^{\mathcal{I}}(y) \\
\geq & \bigvee_{y \in \Delta^{\mathcal{I}}} r^{\mathcal{I}}(g(v), y) \otimes G^{\mathcal{I}}(y) \\
\geq & \bigvee_{y \in \Delta^{\mathcal{I}}} r^{\mathcal{I}}\left(g(v), g\left(\mu^{\prime}\right)\right) \otimes r^{\mathcal{I}}\left(g\left(\mu^{\prime}\right), y\right) \otimes G^{\mathcal{I}}(y) \\
= & r^{\mathcal{I}}\left(g(v), g\left(\mu^{\prime}\right)\right) \otimes(\exists r . G)^{\mathcal{I}}\left(g\left(\mu^{\prime}\right)\right) \\
\geq & s^{\prime \mathcal{I}}\left(g(v), g\left(\mu^{\prime}\right)\right) \otimes(\exists r . G)^{\mathcal{I}}\left(g\left(\mu^{\prime}\right)\right) \\
= & \mathbf{T}\left(\mu^{\prime}\right)(\rho) \otimes \mathbf{T}\left(\mu^{\prime}\right)(\exists r . G),
\end{aligned}
$$

and thus $\mathbf{T}\left(\mu^{\prime}\right)(\exists r . G) \leq \mathbf{T}\left(\mu^{\prime}\right)(\rho) \Rightarrow \mathbf{T}(\nu)(\exists s . G)$.

The claim for $E \in \operatorname{sub}(\mathbf{A}, \mathcal{O})$ of the form $\exists s^{\prime} . F$ or $\forall s^{\prime} . F$ with $s^{\prime} \sqsubseteq \mathcal{R} \bar{s}$ can be shown similarly and for value restrictions $\forall s . G$, analogous arguments can be used.

Finally, if $(C, \ell) \in \mathbf{A}$, then $\mathbf{T}(\varepsilon)(C)=C^{\mathcal{I}}(g(\varepsilon))=$ $C^{\mathcal{I}}(x)=\ell$.

Proof of Lemma 5.2 Consider the Hintikka functions $H$, $H_{0}, \ldots, H_{k}$ and $i, i_{0} \in K$ and let $\left(H_{0}^{\prime}, i_{0}\right):=f_{(H, i)}\left(H_{0}, i_{0}\right)$ and $\left(H_{j}^{\prime}, j\right):=f_{(H, i)}\left(H_{j}, j\right)$ for all $j, 1 \leq j \leq k$. We show that if $\left(H, H_{1}, \ldots, H_{k}\right)$ satisfies the Hintikka condition, then $\left(H, H_{1}^{\prime}, \ldots, H_{k}^{\prime}\right)$ also satisfies it. We show this only for the existential restrictions (Conditions a)-c) of Definition 4.4). The conditions for the value restrictions can be shown by dual arguments.

For Condition a), let $\exists s . G \in \operatorname{sub}(\mathbf{A}, \mathcal{O})$ be such that $H(\exists s . G)$ is defined. Since $\operatorname{rd}_{\mathcal{T}}(G)<\operatorname{rd}_{\mathcal{T}}(\exists s . G) \leq$ $\operatorname{rd}_{\mathcal{T}}(H)$ and $H_{\varphi(\exists s . G)}(G)$ is defined, the value $H_{\varphi(\exists s . G)}^{\prime}(G)$ is also defined and equal to $H_{\varphi(\exists s . G)}(G)$. Moreover, $\operatorname{support}(H) \neq \varnothing$, and thus $H_{\varphi(\exists s . G)}^{\prime}(\rho)=H_{\varphi(\exists s . G)}(\rho)$. This shows that the equality in Condition a) remains satisfied.

To show Condition b), let $\exists s . G \in \operatorname{support}(H)$ and $E \in \operatorname{sub}(\mathbf{A}, \mathcal{O})$ be of the form $\exists s^{\prime} . F$ or $\forall s^{\prime} . F$ with $s^{\prime} \sqsubseteq \mathcal{R} s$. We can show as above that $H_{\varphi(E)}^{\prime}(G)$ and $H_{\varphi(E)}^{\prime}(\rho)$ are defined and equal to $H_{\varphi(E)}(G)$ and $H_{\varphi(E)}(\rho)$, respectively. Thus, the required inequality is still satisfied after applying $f_{(H, s)}$. Since in $L-\mathcal{A} \mathcal{L C H} \mathcal{H}$ there are no transitive roles, the rest of this condition is trivially satisfied. 
For Condition c), let $E \in \operatorname{sub}(\mathbf{A}, \mathcal{O})$ be of the form $\exists s^{\prime} . F$ or $\forall s^{\prime} . F$ with $s^{\prime} \sqsubseteq \mathcal{R} \bar{s}$ and $\exists s . G \in \operatorname{support}\left(H_{\varphi(E)}^{\prime}\right)$. Thus, $H_{\varphi(E)}(\exists s . G)$ is defined and equal to $H_{\varphi(E)}^{\prime}(\exists s . G)$, which implies that $G \in \operatorname{support}(H)$. This in turn implies $\operatorname{support}(H) \neq \varnothing$, which yields $H_{\varphi(E)}^{\prime}(\rho)=H_{\varphi(E)}(\rho)$. This shows that all concerned values are the same as before applying $f_{(H, s)}$, i.e. the inequality is still satisfied.

To show the second condition of Definition 2.3, assume that the tuple $\left(H_{0}, H_{1}, \ldots, H_{k}\right)$ satisfies the Hintikka condition. We show that $\left(H_{0}^{\prime}, H_{1}^{\prime}, \ldots, H_{k}^{\prime}\right)$ also satisfies it.

Let $\exists s . G \in \operatorname{support}\left(H_{0}^{\prime}\right)$. By definition of $f_{(H, s)}$ this implies that $H_{0}(\exists s . G)=H_{0}^{\prime}(\exists s . G)$ and that $\operatorname{rd}_{\mathcal{T}}(G)<$ $\operatorname{rd}_{\mathcal{T}}(\exists s . G)<\operatorname{rd}_{\mathcal{T}}(H)$. Thus, $H_{\varphi(\exists s . G)}(G)$ is also defined and $H_{\varphi(\exists s . G)}^{\prime}(G)=H_{\varphi(\exists s . G)}(G)$. Moreover, $\operatorname{support}(H)$ $\neq \varnothing$, and thus $H_{\varphi(\exists s . G)}^{\prime}(\rho)=H_{\varphi(\exists s . G)}(\rho)$. This shows that Condition a) is still satisfied.

Condition b) can again be shown by similar arguments, replacing $\varphi(\exists s . G)$ by $\varphi(E)$ and the equality condition by an inequality.

For Condition c), let $E \in \operatorname{sub}(\mathbf{A}, \mathcal{O})$ be of the form $\exists s^{\prime} . F$ or $\forall s^{\prime} . F$ with $s^{\prime} \sqsubseteq \mathcal{R} \bar{s}$ and $\exists s . G \in \operatorname{support}\left(H_{\varphi(E)}^{\prime}\right)$. Thus, $H_{\varphi(E)}(\exists s . G)$ must also be defined and equal to $H_{\varphi(E)}^{\prime}(\exists s . G)$. This implies that $H_{0}(G)$ is defined and $\operatorname{rd}_{\mathcal{T}}(G)<\operatorname{rd}_{\mathcal{T}}(\exists s . G)<\operatorname{rd}_{\mathcal{T}}(H)$, and thus $H_{0}^{\prime}(G)$ is also defined and equal to $H_{0}(G)$. Since $\operatorname{support}(H) \neq \varnothing$, we again have $H_{\varphi(E)}^{\prime}(\rho)=H_{\varphi(E)}(\rho)$.

Proof of Lemma 5.5 Consider the Hintikka functions $H$, $H_{0}, \ldots, H_{k}$ and $i, i_{0} \in K$ and let $\left(H_{0}^{\prime}, i_{0}\right):=f_{(H, i)}\left(H_{0}, i_{0}\right)$ and $\left(H_{j}^{\prime}, j\right):=f_{(H, i)}\left(H_{j}, j\right)$ for all $j, 1 \leq j \leq k$. We show that if $\left(H, H_{1}, \ldots, H_{k}\right)$ satisfies the Hintikka condition, then $\left(H, H_{1}^{\prime}, \ldots, H_{k}^{\prime}\right)$ also satisfies it. Once again, we show this only for the existential restrictions.

Condition a) and the first parts of Conditions b) and c) can be shown as for Lemma 5.2. Since in $L-\mathcal{S} \mathcal{I}_{\mathrm{C}}$ the RBox is empty, for the second part of Condition b) we only have to consider the case where $\exists s . G \in \operatorname{support}(H)$, $E \in \operatorname{sub}(\mathbf{A}, \mathcal{O})$ is of the form $\exists s . F$ or $\forall s . F$, and $s$ is transitive. Since in this case $\exists s . G \in \operatorname{support}\left(H_{\varphi(E)}\right)$, it follows that $\exists s .\left.G \in H_{\varphi(E)}\right|_{s}$ and the value $H_{\varphi(E)}^{\prime}(\exists s . G)$ is defined and equal to $H_{\varphi(E)}(\exists s . G)$. As $\operatorname{support}(H) \neq \emptyset$, we also have $H_{\varphi(E)}^{\prime}(\rho)=H_{\varphi(E)}(\rho)$, and thus the required inequality is still satisfied.

For the second part of Condition c), assume that $s$ is transitive, $\exists s . G \in \operatorname{support}\left(H_{\varphi(E)}^{\prime}\right)$ for some $E \in \operatorname{sub}(\mathbf{A}, \mathcal{O})$ of the form $\exists \bar{s} . F$ or $\forall \bar{s} . F$. This implies that $\exists s . G \in \operatorname{sub}^{\leq n-1}(\mathbf{A}, \mathcal{O})$ and the value of $H_{\varphi(E)}(\exists s . G)$ is defined and equal to $H_{\varphi(E)}^{\prime}(\exists s . G)$. By the Hintikka condition, this implies that $\exists s . G \in \operatorname{support}(H)$ and $H_{\varphi(E)}^{\prime}(\rho)=$ $H_{\varphi(E)}(\rho)$, and thus the inequality remains satisfied.

To show the second condition of Definition 2.3, assume that the tuple $\left(H_{0}, H_{1}, \ldots, H_{k}\right)$ satisfies the Hintikka condition. To show that $\left(H_{0}^{\prime}, H_{1}^{\prime}, \ldots, H_{k}^{\prime}\right)$ also satisfies it, we again only consider the second parts of Conditions b) and c); the other conditions follow from previous or dual arguments.

Let $\exists s . G \in \operatorname{support}\left(H_{0}^{\prime}\right), E \in \operatorname{sub}(\mathbf{A}, \mathcal{O})$ be of the form $\exists s . F$ or $\forall s . F$, and $s$ be transitive. This implies that also $H_{0}(\exists s . G)$ is defined and equal to $H_{0}^{\prime}(\exists s . G)$. From the Hintikka condition, it follows that $\exists s . G \in \operatorname{support}\left(H_{\varphi(E)}\right)$. Thus, $H_{\varphi(E)}^{\prime}(\exists s . G)$ is also defined and equal to $H_{\varphi(E)}(\exists s . G)$. Finally, we have $\operatorname{support}(H) \neq \emptyset$, which implies that $H_{\varphi(E)}^{\prime}(\rho)=H_{\varphi(E)}(\rho)$.

Let now $\exists s . G \in \operatorname{support}\left(H_{\varphi(E)}^{\prime}\right)$ for some $E \in \operatorname{sub}(\mathbf{A}, \mathcal{O})$ of the form $\exists \bar{s} . F$ or $\forall \bar{s} . F$ and $s$ be transitive. Thus, $\exists s . G \in \operatorname{sub}^{\leq n-1}(\mathbf{A}, \mathcal{O})$ and the value $H_{\varphi(E)}(\exists s . G)$ is defined and equal to $H_{\varphi(E)}^{\prime}(\exists s . G)$. By the Hintikka condition, we have $\exists s . G \in \operatorname{support}\left(H_{0}\right)$, and thus the value $H_{0}^{\prime}(\exists s . G)$ is also defined and equal to $H_{0}(\exists s . G)$. Finally, the values $H_{\varphi(E)}(\rho)$ and $H_{\varphi(E)}^{\prime}(\rho)$ must also be equal.

Proof of Lemma 5.7 We have to show that the automata $\mathcal{A}_{\mathbf{A}, \mathcal{O}}^{S}$ induced by the functions in Definition 5.4 are polynomially blocking w.r.t. the blocking relation $\longleftarrow \mathcal{S I}_{\mathrm{c}}$. Let $\left(H_{0}, i_{0}\right),\left(H_{1}, i_{1}\right),\left(H_{2}, i_{2}\right)$ be the states of three consecutive nodes in a path of a run of $\mathcal{A}_{\mathrm{A}, \mathcal{O}}^{S}$ and $s_{0}, s_{1}, s_{2}$ be the roles of the restrictions designated by the indices $i_{0}, i_{1}, i_{2}$, respectively. Recall first that the faithful family of functions ensures that

$\operatorname{rd}_{\mathcal{T}}\left(H_{0}\right) \geq \operatorname{rd}_{\mathcal{T}}\left(H_{1}\right) \geq \operatorname{rd}_{\mathcal{T}}\left(H_{2}\right)$.

If $s_{1}$ is not transitive, then $\operatorname{rd}_{\mathcal{T}}\left(H_{0}\right)>\operatorname{rd}_{\mathcal{T}}\left(H_{1}\right)$. Moreover, if $s_{1} \neq s_{2}$, then $\operatorname{rd}_{\mathcal{T}}\left(H_{0}\right)>\operatorname{rd}_{\mathcal{T}}\left(H_{2}\right)$, regardless of whether $s_{1}, s_{2}$ are transitive or not. Thus, a path in a run can have at most

$\max \left\{\operatorname{rd}_{\mathcal{T}}(D) \mid D \in \operatorname{sub}(\mathbf{A}, \mathcal{O})\right\}+1$

states using a non-transitive role, or using different roles for consecutive transitions before reaching a state $(H, s)$ with $\operatorname{support}(H)=\emptyset$.

If $s_{1}=s_{2}$ is a transitive role, then the role depth of the Hintikka functions may remain constant through both transitions. From the Hintikka condition, $\left.\left.\mathrm{H}_{1}\right|_{s} \subseteq \mathrm{H}_{2}\right|_{s}, \mathrm{H}_{1}^{-s} \subseteq \mathrm{H}_{2}^{-s}$, and $\left.\left.H_{2}\right|_{\bar{s}} \subseteq H_{1}\right|_{\bar{s}}$ must hold. This means that there can be at most $|\operatorname{sub}(\mathbf{A}, \mathcal{O})|$ states $(H, s)$ with $H(\rho)=\mathbf{0}$ before Condition (ii) of the blocking relation triggers.

Finally, if $H_{1}(\rho)=\mathbf{1}$, the Hintikka condition implies that $H_{0}(\forall s . G) \leq H_{1}(\rho) \Rightarrow H_{1}(\forall s . G)=H_{1}(\forall s . G)$

for any $\forall s . G \in \operatorname{support}\left(H_{0}\right)$, and dually

$H_{0}(\exists s . G) \leq H_{1}(\rho) \otimes H_{1}(\exists s . G)=H_{1}(\exists s . G)$

whenever $\exists s . G \in \operatorname{support}\left(H_{0}\right)$. This means that after a chain of at most $|L||\operatorname{sub}(\mathbf{A}, \mathcal{O})|$ transitions with role $s$ to degree $\mathbf{1}$, we find two states $(H, s),\left(H^{\prime}, s\right)$ such that $H(D)=H^{\prime}(D)$ for every $D$ in $\mathcal{Q}\left(H, H_{i}, s\right)$. The Hintikka condition ensures that $H_{0}(\forall s . G) \leq H_{1}(\rho) \Rightarrow H_{1}(G)=$ $H_{1}(G)$ and $H_{0}(\exists s . G) \leq H_{1}(G)$, which shows that the last 
condition is also satisfied after at most $|L||\operatorname{sub}(\mathbf{A}, \mathcal{O})|$ transitions.

An additional factor of $|L||\operatorname{sub}(\mathbf{A}, \mathcal{O})|$ enables us to ensure the existence two nodes $(H, i)$ and $\left(H^{\prime}, i^{\prime}\right)$ that satisfy the remaining condition of $\longleftarrow \mathcal{S} \mathcal{I}_{c}$, namely that $i=i^{\prime}=\varphi(E)$ for some $E \in \operatorname{sub}(\mathbf{A}, \mathcal{O})$ of the form $\exists s . F$ or $\forall s . F$ and that $H(F)=H^{\prime}(F)$.

In total, every path of length at least $(|L||\operatorname{sub}(\mathbf{A}, \mathcal{O})|)^{5}$ will contain two nodes that are in the blocking relation. This number is obviously polynomial in the size of $\mathbf{A}, \mathcal{O}$.

Proof of Lemma 6.1 If an interpretation $\mathcal{I}$ satisfies the concept definition $\langle A \doteq C \sqcap D, \ell\rangle$, then we have $A^{\mathcal{I}}(x) \Rightarrow\left(C^{\mathcal{I}}(x) \otimes D^{\mathcal{I}}(x)\right) \geq \ell$, and thus $C^{\mathcal{I}}(x) \geq C^{\mathcal{I}}(x) \otimes D^{\mathcal{I}}(x) \geq A^{\mathcal{I}}(x) \otimes \ell$, for every $x \in \Delta^{\mathcal{I}}$. This implies that $A^{\mathcal{I}}(x) \Rightarrow C^{\mathcal{I}}(x) \geq \ell$ holds for all $x \in \Delta^{\mathcal{I}}$, i.e. $\mathcal{I}$ satisfies the GCI $\langle A \sqsubseteq C, \ell\rangle$.

Assume now that $\mathcal{I}$ satisfies $\langle A \sqsubseteq C, \ell\rangle$ and let $x \in \Delta^{\mathcal{I}}$. We extend $\mathcal{I}$ to the new concept name $D$ by defining $D^{\mathcal{I}}(x):=C^{\mathcal{I}}(x) \Rightarrow A^{\mathcal{I}}(x)$. We will first show the following claim, which generalizes a well-known result for continuous t-norms over the real interval $[0,1]$ and implies that $C^{\mathcal{I}}(x) \otimes D^{\mathcal{I}}(x)=A^{\mathcal{I}}(x) \wedge C^{\mathcal{I}}(x):$

Claim If $\ell, m \in L$, then $\ell \otimes(\ell \Rightarrow m)=\ell \wedge m$.

Proof Since $\otimes$ is monotone, we have $\ell \otimes(\ell \Rightarrow m) \leq \ell$, and by continuity of $\otimes$, we get $\ell \otimes(\ell \Rightarrow m)=$ $\bigvee\{\ell \otimes x \mid \ell \otimes x \leq m\} \leq m$. Let now $x \in L$ be such that $x \leq \ell$ and $x \leq m$. Then $\ell \otimes x \leq x \leq m$, and thus $x \leq \ell \Rightarrow m$. By continuity of $\otimes$, these facts imply that

$$
\begin{aligned}
x \vee(\ell \otimes(\ell \Rightarrow m)) & =(x \vee \ell) \otimes(x \vee(\ell \Rightarrow m)) \\
& =\ell \otimes(\ell \Rightarrow m),
\end{aligned}
$$

which shows that $x \leq \ell \otimes(\ell \Rightarrow m)$.

We now have

$$
\begin{aligned}
& \left(C^{\mathcal{I}}(x) \otimes D^{\mathcal{I}}(x)\right) \Rightarrow A^{\mathcal{I}}(x) \\
& \quad=\left(A^{\mathcal{I}}(x) \wedge C^{\mathcal{I}}(x)\right) \Rightarrow A^{\mathcal{I}}(x)=\mathbf{1} .
\end{aligned}
$$

Since $\mathcal{I}$ satisfies $\langle A \sqsubseteq C, \ell\rangle$, we have $A^{\mathcal{I}}(x) \Rightarrow C^{\mathcal{I}}(x) \geq \ell$, and thus $C^{\mathcal{I}}(x) \geq \bar{A}^{\mathcal{I}}(x) \otimes \ell$. Furthermore, we know that $A^{\mathcal{I}}(x) \geq A^{\mathcal{I}}(x) \otimes \ell$, which implies that

$C^{\mathcal{I}}(x) \otimes D^{\mathcal{I}}(x)=A^{\mathcal{I}}(x) \wedge C^{\mathcal{I}}(x) \geq A^{\mathcal{I}}(x) \otimes \ell$,

and thus

$A^{\mathcal{I}}(x) \Rightarrow\left(C^{\mathcal{I}}(x) \otimes D^{\mathcal{I}}(x)\right) \geq \ell$.

Together, Eqs. (1) and (2) imply that $\mathcal{I}$ satisfies the concept definition $\langle A \doteq C \sqcap D, \ell\rangle$ at $x$.

\section{Appendix B: Examples}

Example B.1 We now provide a simple stepwise construction of an automaton giving a PSPACE upper bound to reasoning. Suppose we want to determine the complexity of the

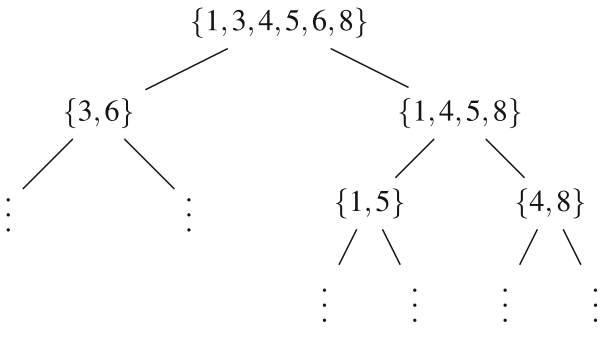

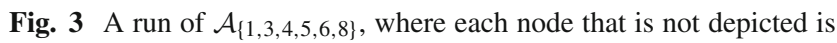
assigned an arbitrary state of cardinality 2

following number theoretical problem: given a finite set $N$ of positive integers, can it be partitioned into two subsets $A_{1}, A_{2}$ such that $2 \sum A_{1}=\sum A_{2}$, and each $A_{1}$ and $A_{2}$ can also be recursively partitioned in this way, unless the cardinality of the set is smaller than 3 ? This problem can be solved by deciding the emptiness of the looping automaton $\mathcal{A}_{N}=\left(Q_{N}, I_{N}, \Delta_{N}\right)$ over binary trees, where

$$
\begin{aligned}
-Q_{N} & :=\mathcal{P}(N), \\
- & I_{N}:=\{N\}, \text { and } \\
-\Delta_{N}:=\{(A, B, C)|| A \mid \leq 2 \text { or } B \cap C=\emptyset, & \\
& \left.B \cup C=A, 2 \sum B=\sum C\right\} .
\end{aligned}
$$

Figure 3 depicts a run of $\mathcal{A}_{N}$ for the input $N=\{1,3,4,5$, $6,8\}$. It is clear that such an automaton has a run iff the problem stated above has a solution. The number of states is exponential in the size of $N$. Since emptiness of looping automata can be decided in PTIME, this problem is in EXPTIME.

However, it is easy to see that the following family of functions $f_{A}: Q_{N} \rightarrow Q_{N}$ for $A \in Q_{N}$ is faithful w.r.t. $\mathcal{A}_{N}$ :

$f_{A}\left(A^{\prime}\right):=\left\{\begin{array}{ll}A^{\prime} & \text { if }|A|>2 \\ \varnothing & \text { if }|A| \leq 2\end{array}\right.$.

The example run from Fig. 3 can easily be transformed into a run of the induced subautomaton $\mathcal{A}_{N}^{S}$ by labeling the nodes that are not depicted by $\emptyset$.

Furthermore, we can show that the construction of $\mathcal{A}_{N}^{S}$ from the input $N$ is a PSPACE on-the-fly construction:

(i) With equality as the blocking relation, $\mathcal{A}_{N}^{S}$ is $(|N|+1)$ blocking since every transition must reduce the cardinality of the set by at least 1 , and thus after at most $|N|$ transitions the empty set must be reached. The arity of $\mathcal{A}_{N}^{S}$ is always constant (2).

(ii) Every element of $Q_{N}$ has size polynomial in the size of $N$.

(iii) We do not have to guess elements of $I_{N}$ since it contains only one element that is clearly of size polynomial in the size of $N$. For a given state $A \in Q_{N}$ of cardinality greater than 2 one can guess a partition of $A$ into two subsets $B, C$ and check whether they satisfy the conditions of $\Delta_{N}$ in polynomial time. 


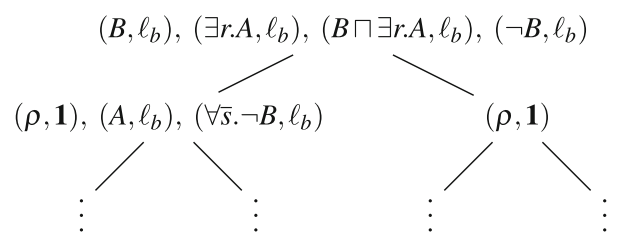

Fig. 4 A run of $\mathcal{A}_{\mathbf{A}, \mathcal{O}}$ showing the defined concept-value-pairs of the Hintikka functions in the states. Each node that is not depicted only assigns 1 to $\rho$

This shows that the problem is also in PSPACE. This example illustrates that a naive modeling of a problem using looping automata can be easy to describe, but might not yield a good complexity bound. By a subsequent faithful reduction to a PSPACE on-the-fly-construction, the complexity bound can be improved.

Example B.2 Consider the lattice $L_{2}$ from Fig. 2 with the Gödel $t$-norm, the ontology $\mathcal{O}=(\mathcal{T}, \mathcal{R})$, where $\mathcal{T}=$ $\{\langle A \doteq \forall \bar{s} . \neg B, \mathbf{1}\rangle\}$ and $\mathcal{R}=\{r \sqsubseteq s\}$, and the local ABox $\mathbf{A}=\left\{\left(B \sqcap \exists r . A, \ell_{b}\right)\right\}$. The set of subconcepts $\operatorname{sub}(\mathbf{A}, \mathcal{O})$ consists of the elements $A, B, \exists r . A, \neg B, \forall \bar{s} . \neg B$, and $B \sqcap \exists r . A$. The arity $k$ of the Hintikka automaton is 2 since we only have the restrictions $\exists r . A$ and $\forall \bar{s} . \neg B$. We fix the mapping $\varphi$ by setting $\varphi(\exists r . A)=1$ and $\varphi(\forall \bar{s} . \neg B)=2$.

Figure 4 depicts the beginning of a run of the Hintikka automaton $\mathcal{A}_{\mathbf{A}, \mathcal{O}}$. The Hintikka function $H_{0}$ labeling the root must be an initial state, i.e. we must have $H_{0}(B \sqcap \exists r . A)=\ell_{b}$. Definition 4.3 forces us to assign to $B$ and $\exists r$.A two values whose infimum is $\ell_{b}$. Here, we guess $H_{0}(B)=H_{0}(\exists r . A)=\ell_{b}$. Thus, the Hintikka function $H_{1}$ labeling the first successor must satisfy Definition 4.4a), which implies that $H_{1}(\rho) \otimes H_{1}(A)$ must be $\ell_{b}$. If we guess $H_{1}(A)=\ell_{b}$, the compatibility condition (see Definition 4.3) requires that $H_{1}(\forall \bar{s} . \neg B)$ is also $\ell_{b}$. Finally, since $r \sqsubseteq \mathcal{R} \overline{\bar{s}}$, Definition $\left.4.4 c^{\prime}\right)$ requires that

$H_{1}(\forall \bar{s} . \neg B) \leq H_{1}(\rho) \Rightarrow H_{0}(\neg B)$.

This can be satisfied by setting $H_{0}(\neg B)$ to $\ell_{b}$. This assignment satisfies Definition 4.3 since $H_{0}(\neg B)=\ell_{b}=\sim \ell_{b}=$ $\sim H_{0}(B)$.

All other requirements of Definitions 4.3 and 4.4 are trivially satisfied, and thus $\mathbf{A}$ is locally consistent w.r.t. $\mathcal{O}$. On the other hand, $\{(B \sqcap \exists r . A, \mathbf{1})\}$ is not locally consistent w.r.t. $\mathcal{O}$ since we cannot assign $\mathbf{1}$ to both $B$ and $\neg B$ at the root.

\section{References}

1. Baader F, Calvanese D, McGuinness DL, Nardi D, Patel-Schneider PF (eds) (2007) The description logic handbook: theory, implementation, and applications, 2nd edn. Cambridge University Press, Cambridge
2. Baader F, Hladik J, Peñaloza R (2008) Automata can show PSPACE results for description logics. Inf Comput 206(9-10):1045-1056. doi:10.1016/j.ic.2008.03.006

3. Baader F, Peñaloza R (2011) Are fuzzy description logics with general concept inclusion axioms decidable? In: Proceedings of the 2011 IEEE international conference on fuzzy systems (FUZZIEEE'11), pp 1735-1742. IEEE Computer Society Press, New York. doi:10.1109/FUZZY.2011.6007520

4. Baader F, Peñaloza R (2011) On the undecidability of fuzzy description logics with GCIs and product $t$-norm. In: Tinelli C, Sofronie-Stokkermans V (eds) Proceedings of the 8th international symposium on frontiers of combining systems (FroCoS'11). Lecture notes in computer science, vol 6989. Springer, Berlin, pp 55-70. doi:10.1007/978-3-642-24364-6_5

5. Belnap ND (1977) A useful four-valued logic. In: Epstein G, Dunn JM (eds) Modern uses of multiple-valued logic. Reidel Publishing Company, Dordrecht, pp 7-37

6. Bobillo F, Bou F, Straccia U (2011) On the failure of the finite model property in some fuzzy description logics. Fuzzy Sets Syst 172(1):1-12. doi:10.1016/j.fss.2011.02.012

7. Bobillo F, Delgado M, Gómez-Romero J, Straccia U (2012) Joining gödel and zadeh fuzzy logics in fuzzy description logics. Int J Uncertainty Fuzziness Knowl Based Syst 20(4):475-508

8. Bobillo F, Straccia U (2007) A fuzzy description logic with product $t$-norm. In: Proceedings of the 2007 IEEE international conference on fuzzy systems (FUZZ-IEEE'07). IEEE Computer Society Press, New York, pp 1-6: doi:10.1109/FUZZY.2007.4295443

9. Bobillo F, Straccia U (2009) Fuzzy description logics with general $t$-norms and datatypes. Fuzzy Sets Syst 160(23):3382-3402. doi:10.1016/j.fss.2009.03.006

10. Bobillo F, Straccia U (2010) Finite fuzzy description logics: a crisp representation for finite fuzzy $\mathcal{A L C H}$. In: Bobillo F, Carvalho R, da Costa PCG, D'Amato C, Fanizzi N, Laskey KB, Laskey KJ, Lukasiewicz T, Martin T, Nickles M, Pool M (eds) Proceedings of the 6th international workshop on uncertainty reasoning for the semantic web (URSW'10), CEUR Workshop Proceedings, vol 654, pp 61-72. http://ceur-ws.org/Vol-654/paper6.pdf

11. Bobillo F, Straccia U (2011) Reasoning with the finitely manyvalued Łukasiewicz fuzzy description logic $\mathcal{S R O I} \mathcal{L}$. Inf Sci 181:758-778. doi:j.ins.2010.10.020

12. Borgwardt S, Peñaloza R (2011) Description logics over lattices with multi-valued ontologies. In: Walsh $\mathrm{T}$ (ed) Proceedings of the 22nd international joint conference on artifical intelligence (IJCAI'11). AAAI Press, Menlo Park, pp 768-773. doi:10.5591/ 978-1-57735-516-8/IJCAI11-135

13. Borgwardt S, Peñaloza R (2011) Finite lattices do not make reasoning in $\mathcal{A L C I}$ harder. In: Bobillo $\mathrm{F}$, Carvalho $\mathrm{R}$, da Costa PCG, d'Amato C, Fanizzi N, Laskey KB, Laskey KJ, Lukasiewicz T, Martin T, Nickles M, Pool M (eds) Proceedings of the 7th international workshop on uncertainty reasoning for the semantic web (URSW'11), CEUR workshop proceedings, vol 778, pp 51-62. http://ceur-ws.org/Vol-778/paper5.pdf

14. Borgwardt S, Peñaloza R (2011) Fuzzy ontologies over lattices with $t$-norms. In: Rosati R, Rudolph S, Zakharyaschev M (eds) Proceedings of the 2011 international workshop on description logics (DL'11), CEUR Workshop Proceedings, vol 745, pp 70-80. http:// ceur-ws.org/Vol-745/paper_1.pdf

15. Borgwardt S, Peñaloza R (2012) A tableau algorithm for fuzzy description logics over residuated De Morgan lattices. In: Krötzsch M, Straccia U (eds) Proceedings of the 6th international conference on web reasoning and rule systems (RR'12), Lecture notes in computer science, vol 7497. Springer, Berlin, pp 9-24. doi:10.1007/ 978-3-642-33203-6_3

16. Borgwardt S, Peñaloza R (2012) Undecidability of fuzzy description logics. In: Brewka G, Eiter T, McIlraith SA (eds) Proceedings of the 13th international conference on principles of knowledge 
representation and reasoning (KR 2012). AAAI Press, Rome, pp 232-242

17. Bou F, Cerami M, Esteva F (2011) Finite-valued Łukasiewicz modal logic is PSPACE-complete. In: Walsh T (ed) Proceedings of the 22nd international joint conference on artifical intelligence (IJCAI'11). AAAI Press, Menlo Park, pp 774-779. doi:10.5591/ 978-1-57735-516-8/IJCAI11-136

18. Cerami M, Straccia U (2011) On the undecidability of fuzzy description logics with GCIs with Łukasiewicz $t$-norm . http:// arxiv.org/abs/1107.4212

19. De Cooman G, Kerre EE (1993) Order norms on bounded partially ordered sets. J Fuzzy Math 2:281-310

20. Goguen JA (1967) L-fuzzy sets. J Math Anal Appl 18(1):145-174. doi:10.1016/0022-247X(67)90189-8

21. Grätzer G (2003) General lattice theory, 2nd edn. Birkhäuser, Basel

22. Hájek P (2001) Metamathematics of fuzzy logic (trends in logic). Springer, Berlin

23. Hájek P (2005) Making fuzzy description logic more general. Fuzzy Sets Syst 154(1):1-15. doi:10.1016/j.fss.2005.03.005

24. Horrocks I, Sattler U (1999) A description logic with transitive and inverse roles and role hierarchies. J Logic Comput 9(3):385-410. doi:10.1093/logcom/9.3.385

25. Horrocks I, Sattler U, Tobies S (2000) Practical reasoning for very expressive description logics. J Int Group Pure Appl Logic 8(3):239-263. doi:10.1093/jigpal/8.3.239

26. Lukasiewicz T, Straccia U (2008) Managing uncertainty and vagueness in description logics for the semantic web. J Web Seman 6(4):291-308. doi:10.1016/j.websem.2008.04.001
27. Molitor R, Tresp CB (2000) Extending description logics to vague knowledge in medicine. In: Szczepaniak PS, Lisboa PJG, Kacprzyk $\mathbf{J}$ (eds) Fuzzy systems in medicine, studies in fuzziness and soft computing, vol 41. Springer, Berlin, pp 617-635. http://www. springer.com/public+health/book/978-3-7908-1263-3

28. Schild K (1991) A correspondence theory for terminological logics: preliminary report. In: Mylopoulos J, Reiter R (eds) Proceedings of the 12th international joint conference on artificial intelligence (IJCAI'91). Morgan Kaufmann, San Francisco, pp 466-471. http:// ijcai.org/Past\%20Proceedings/IJCAI-91-VOL1/PDF/072.pdf

29. Schmidt-Schauß M, Smolka G (1991) Attributive concept descriptions with complements. Artif Intell 48(1):1-26. doi:10.1016/ 0004-3702(91)90078-X

30. Straccia U (2006) Description logics over lattices. Int J Uncertainty Fuzziness Knowl Based Syst 14(1):1-16. doi:10.1142/ S0218488506003807

31. Tobies S (2001) Complexity results and practical algorithms for logics in knowledge representation. Ph.D. thesis, RWTH Aachen. http://lat.inf.tu-dresden.de/research/phd/\#Tobies-PhD-2001

32. Vardi MY, Wolper P (1986) Automata-theoretic techniques for modal logics of programs. J Comput Syst Sci 32(2):183-221. doi:10.1016/0022-0000(86)90026-7

33. Zadeh LA (1965) Fuzzy sets. Inf. Control 8(3):338-353

34. Zherui M, Wangming W (1991) Logical operators on complete lattices. Inf Syst 55(1-3):77-97. doi:10.1016/0020-0255(91)90007$\mathrm{H}$ 